\title{
Gegenspieler, Vetospieler oder was? Demokratiefunktionales Agieren des Bundesverfassungsgerichts 1951-2005
}

\author{
Sascha Kneip
}

Opponent, Veto Player, or what? Functional and Democratic Acting of the Federal Constitutional Court 1951-2005

Abstract: Constitutional courts are considered powerful opponents of democratic politics and are seen as key actors in processes of judicialization. The paper discusses both assumptions in theoretical and empirical terms. In theoretical terms, it calls for expanding the "opponents thesis" with a democratic theory perspective. Empirically, it shows that the German Federal Constitutional Court has neither been an antagonist of democratic politics nor actively pursued processes of judicialization. Instead, the court has met adequately its functional role in the democratic governmental system.

Keywords: Constitutional Courts, Constitutionalism, Judicialization, Democratic Theory

Schlagwörter: Verfassungsgerichtsbarkeit, Konstitutionalismus, Justizialisierung, Demokratietheorie

\section{Einleitung}

Dass Verfassungsgerichte mächtige (Mit-)Spieler demokratischer Politik (geworden) sind, darf heute als allgemein geteilte Überzeugung gelten (siehe statt vieler nur Waltman u. Holland 1988; Vallinder 1995; Stone Sweet 2000; Epstein et al. 2001; Guarnieri u. Pederzoli 2002; Ginsburg 2003; Koopmans 2003; Sandler u. Schoenbrod 2003; Epstein u. Knight 2004; Scheppele 2005; Horowitz 2006; Romeu 2006). Das 20. Jahrhundert muss insofern nicht nur als ein Zeitalter der Demokratisierungsprozesse, sondern ebenso als eine Epoche der Konstitutionalisierung von Politik begriffen werden (Elster u. Slagstad 1993; Henkin 1994; Holmes 1995; Bellamy 1996; Alexander 1998; Hilbink 2008). Besaßen im Jahr 1920 nur eine Handvoll Staaten eine etablierte (diffuse oder spezialisierte) Verfassungsgerichtsbarkeit, lässt sich heute kaum eine Demokratie finden, die kein Verfassungsgericht der einen oder anderen Art institutionalisiert hat. Die Bindung der Politik durch Verfassungen und Grundrechte gehört heute ebenso zum Selbstbild moderner Demokratien wie die Sicherung der Grundrechte durch Verfassungsgerichte. Selbst die ,Mutter der Parlamentssouveränität‘, Großbritannien, hat im Jahr 2009 ein Oberstes Gericht eingerichtet, das zwar nach wie vor keine Normenkontrollkompetenz besitzt, das aber dessen ungeachtet Exekutivakte am Maßstab der Gesetze überprüfen und sanktionieren kann. Und auch in Großbritannien weicht die informelle Selbstbindung der Politik an Grundrechte und -normen zusehends einer formellen Bindung durch institutionalisierte rechtsstaatliche Verfahren. Der im Jahr 2000 implementierte Human Rights Act hat den Grund- 
stein für ein System der Verfassungskontrolle gelegt, dessen Auswirkungen erst allmählich sichtbar werden (vgl. Kavanagh 2009). ${ }^{1}$ Kurzum: Verfassungsgerichte und verfassungsgerichtliche Kontrolle von Legislativ- und/oder Exekutivakten sind heute aus dem Leben etablierter Demokratien nicht mehr wegzudenken.

Aus der empirischen Tatsache ihrer Existenz folgt jedoch noch keine normative oder faktische Akzeptanz ihrer Tätigkeit. Weder die politisch Handelnden noch die Wissenschaft teilen notwendigerweise die Einschätzung, dass Verfassungsgerichte für Demokratien unverzichtbar seien. Im Gegenteil: Sowohl in der normativen als auch in der empirisch ausgerichteten Demokratieforschung macht sich mitunter Skepsis gegenüber Status, Funktion und Rolle von Verfassungsgerichten breit. Während Teile der normativen Demokratie- und Rechtstheorie grundsätzlich die rechtliche Einhegung demokratischer Verfahren kritisch betrachten (z. B. Maus 1992, 2004, 2005; Waldron 1999, 2006), hegt die empirisch ausgerichtete Forschung mitunter den Verdacht, Verfassungsgerichte mischten sich - als demokratisch zu schwach, da nur mittelbar legitimierte Akteure - zu sehr in die Belange „der Politik“ ein (vgl. statt vieler Landfried 1984, 1985, 1994; Höffe 1999; Schneider 1999; Scholz 1999). Beide Perspektiven verknüpfen ihre jeweiligen Beobachtungen häufig mit drei generellen Einschätzungen:

Erstens wird behauptet, Verfassungsgerichte seien „Veto-“ oder „Gegenspieler“ der Politik (Alivizatos 1995; Magaloni u. Sánchez 2001; Volcansek 2001; Tsebelis 2002; Wagschal 2006), „Nebenregierungen“ (Schmidt 2002), „Ersatzgesetzgeber“ (Scholz 1999; Blasberg 2003) oder gar „dritte Parlamentskammern“ (Stone 1992). Diese Einschätzung gründet sich zum einen auf der Analyse der formalen Macht und des institutionalisierten Einflusses von Verfassungsgerichten (vgl. Alivizatos 1995; Lijphart 1999; Tsebelis 2002), zum anderen auf der spezifischeren Betrachtung der z. T. umfangreichen Normenkontrollmöglichkeiten eines Verfassungsgerichts. Aus der formalen institutionellen Stärke von Verfassungsgerichten im Allgemeinen sowie der spezifischen Kompetenz zum judicial review wird gefolgert, dass Gerichte ,Gegenspieler' der Regierung und des Parlaments seien.

Zweitens scheint sich die These, dass Verfassungsgerichte die treibende Kraft hinter einer zunehmenden Justizialisierung der Politik seien, zu einem der dominierenden Argumente innerhalb der sozialwissenschaftlichen Literatur zu Rechtsstaatlichkeit und Demokratie entwickelt zu haben (so jedenfalls Hönnige u. Gschwend 2010, S. 509). Die Vorstellung, dass das Handeln politischer Akteure durch die Existenz und die Tätigkeit eines Verfassungsgerichts eingeschränkt wird, mutet zunächst überaus plausibel an und ist auch in diversen Studien überprüft worden (siehe u. a. Landfried 1984, 1994; Vallinder 1994; Tate u. Vallinder 1995; Stone Sweet 1999, 2000; Shapiro u. Stone Sweet 2002). Allerdings wird häufig Unterschiedliches unter „Justizialisierung“ verstanden, sodass genauer de-

1 Zudem darf nicht vergessen werden, dass Großbritannien - wie auch die Niederlande oder die Schweiz als weitere Beispiele demokratischer Staaten ohne ausgeprägte Verfassungsgerichtsbarkeit - ohnehin in ein europäisches Mehrebenen-Verfassungsgerichtssystem eingebunden ist und sich der Rechtsprechung des Europäischen Gerichtshofs (EuGH) bzw. des Europäischen Gerichtshofs für Menschenrechte (EMRGH) unterworfen hat. Die Vorstellung, demokratisches Regieren sei in diesen Staaten nicht durch rechtsstaatliche Institutionen eingehegt, ist von der Rechtswirklichkeit längst überholt worden. 
finiert werden müsste, wer oder was genau ,justizialisiert' wird, um genauere empirische Aussagen über ihren Umfang geben zu können (mehr dazu weiter unten).

Drittens schwingt in beiden Beobachtungsperspektiven ein normativer Subtext mit, der zwar nicht immer offen formuliert wird (siehe aber z. B. Sandler u. Schoenbrod 2003; Hirschl 2007), der aber latent die Analysen von Verfassungsgerichtshandeln zu begleiten scheint - die Befürchtung nämlich, dass verfassungsgerichtliches Agieren ,schlecht' oder ,nachteilig' für demokratisches Regieren sei, weil entweder ,zu häufig' und ,zu viel' von demokratisch schwach legitimierten Richterinnen und Richtern entschieden werde und die ,eigentlich' demokratische Instanz - das Volk, das Parlament oder die Exekutive - zu wenige Handlungsspielräume zurückbehalte, oder weil die Entscheidungen von Verfassungsgerichten zu inhaltlich unerwünschten Ergebnissen führten.

Alle drei Einschätzungen muten auf den ersten Blick plausibel an. Sie sind aber bislang in den seltensten Fällen einer empirischen Überprüfung unterzogen worden. Die stärkste empirische Basis kann sicher die Justizialisierungsthese für sich beanspruchen, da sie in diversen Einzelfallstudien untersucht worden ist (s. o.). Die „Gegenspielerthese“ wie die These eines „undemokratischen Impacts“ von Verfassungsgerichten hingegen sind - abgesehen von Analysen einzelner aufsehenerregender Urteile wie zum Beispiel des „Kruzifixurteils“ des Bundesverfassungsgerichts (siehe statt vieler z. B. Hufen 1998; Höffe 1999; Schaal 2006) - bislang kaum empirisch untersucht worden. Der vorliegende Beitrag möchte daher anhand einer empirischen Analyse des Outputs des Bundesverfassungsgerichts zwischen den Jahren 1951 und 2005 die hier aufgeworfenen Fragen und Einwände diskutieren und die Tragfähigkeit der diskutierten Annahmen überprüfen. Er will damit dazu beitragen, aus dem „unbekannten Wesen“ Bundesverfassungsgericht (Hönnige u. Gschwend 2010) einen ,guten Bekannten` zu machen - und die Diskussion über die Leistung von Verfassungsgerichten für Demokratien um empirische Erkenntnisse bereichern (siehe zum Bundesverfassungsgericht auch die Beiträge in Vorländer 2006 und Ooyen u. Möllers 2006 sowie die jüngsten Studien von Gawron u. Rogowski 2007; Hönnige 2007; Lembcke 2007; Steinsdorff 2009; Kranenpohl 2010).

Dies soll in drei Schritten geschehen. In einem ersten Schritt soll zunächst kurz diskutiert werden, was genau unter "Justizialisierung“ der Politik und dem Vetospielerstatus eines Verfassungsgerichts verstanden werden kann. Im zweiten Schritt, dem zentralen empirischen Teil des Papiers, werden anschließend die Ergebnisse einer Längsschnittstudie zum Bundesverfassungsgericht vorgestellt und diskutiert, um in einem dritten Schritt schließlich die Frage beantworten zu können, inwieweit das Bundesverfassungsgericht - und Verfassungsgerichte im Allgemeinen - Veto- oder Gegenspieler der Politik sind - und ob sie, unter demokratiefunktionalen Gesichtspunkten, der Demokratie eher ,gut ${ }^{6}$ oder ,schlecht ${ }^{6}$ tun. 


\section{Die Justizialisierung der Politik und der Vetospielerstatus der Verfassungsge- richtsbarkeit}

In ihrer allgemeinen Fassung besagt die These der Justizialisierung der Politik zunächst nichts anderes, als dass politisches Handeln durch das Agieren von Verfassungsgerichten in der einen oder anderen Art eingehegt, begrenzt oder beschränkt wird. Zwei prinzipielle Varianten dieser These sind denkbar (vgl. auch Hönnige u. Gschwend 2010, S. 509-511): Die erste Variante besagt, dass Verfassungsgerichte durch ihr Agieren Handlungsmöglichkeiten der Politik aktiv einschränken (weshalb man diese Variante auch „aktive Justizialisierung“ nennen könnte). Durch ihre Urteile, so die Argumentation, definieren (Verfassungs-)Gerichte autoritativ, welche Verhaltensanforderungen die Verfassung an Bürger und politisch Handelnde stellt und welche Politikinhalte verfassungsgemäß umgesetzt werden können (und welche nicht). Dabei bleiben die Gerichte häufig nicht bei der bloßen Auslegung der Verfassung stehen, sondern sie interpretieren den Verfassungstext nach je eigenen dogmatischen Auslegungsmethoden und Prinzipien und schreiben ihm dadurch Inhalte zu, die nicht ohne Weiteres aus dem Text selbst erkennbar sind. Folge dieses Handelns - und der insgesamt hohen Anzahl von Verfassungsgerichtsurteilen - ist, dass sich die tatsächlichen Handlungsmöglichkeiten der Politik mehr und mehr einengen, ohne dass , die Politik' diesseits der Verfassungsänderung darauf reagieren könnte. Je mehr Urteile in einem bestimmten Politikbereich ergehen, so die Annahme, desto geringer ist ceteris paribus der Handlungsraum für politische Akteure, da die Summe der Urteile die Handlungsalternativen implizit oder explizit begrenzt.

Die zweite Variante der Justizialisierungsthese beruht auf der Analyse der Selbstbeschränkung - oder Autolimitation - politischer Akteure im Angesicht möglicher oder tatsächlicher Verfassungsgerichtsurteile. Rechnen etwa Regierungen oder Parlamentsmehrheiten damit, dass die von ihnen präferierte Politik über Verfassungsklagen angegriffen und für verfassungswidrig erklärt werden könnte, schrecken sie, so die Annahme, mitunter von vornherein vor der Implementierung ihrer Politik zurück, obwohl sie selbst wie auch ihre Wähler von ihrer Richtigkeit überzeugt sind - sie beschränken sich also im Angesicht möglicher juristischer Niederlagen selbst und sorgen damit paradoxerweise für eine Art „vorauseilende Justizialisierung “ der Politik ohne aktives Zutun der Justiz selbst (diese Variante könnte in Abgrenzung zur ersten als „passive Justizialisierung“ bezeichnet werden). Die Hauptursache für diese Form der Justizialisierung ist in der Offenheit des Gerichtszugangs zu suchen (vgl. Vallinder 1994; Tate u. Vallinder 1995; Stone Sweet 1999): Je mehr Akteuren (Gerichten, Opposition, Bürgern) die prinzipielle Möglichkeit eröffnet wird, Normen und Handlungen durch Verfassungsgerichte überprüfen zu lassen, umso mehr sind politische Akteure gezwungen, dies bei der Verabschiedung von Gesetzen zu berücksichtigen. Diese passive Justizialisierung konnte in diversen Einzelfallstudien nachgezeichnet werden, für die Bundesrepublik insbesondere in den Arbeiten von Christine Landfried (siehe z. B. Landfried 1984, 1985, 1992, 1994).

Je mehr Akteure also die Möglichkeit erhalten, Normen oder Handlungen vor Gericht überprüfen zu lassen, so die weithin geteilte Annahme, und je einfacher 
dies institutionell möglich ist, desto größere Justizialisierungseffekte sind zu erwarten (klassisch: Stone Sweet 2000, der insbesondere auf die Rolle oppositioneller Klagen abhebt). Diese Sichtweise greift jedoch zu kurz: Häufigere Klagen müssen nicht per se auch häufigere Interventionen durch Verfassungsgerichte nach sich ziehen - und damit auch nicht notwendigerweise eine stärkere Justizialisierung. Denkbar wäre ebenso, dass ein Gericht trotz häufigerer Aktivierung eine einmal gewählte Zurückhaltung aufrechterhält und dadurch nicht stärker in die Politik ,hineinregiert', wie sich beispielsweise am japanischen Supreme Court beobachten lässt (Itoh 1990; Ramseyer 1991; Ramseyer u. Rasmusen 2003; Haley 2005). Sinnvollerweise sollten noch andere Gründe für Justizialisierungsprozesse (oder ihr Ausbleiben) in Betracht gezogen werden, insbesondere etwa das Rollenverständnis eines Gerichts, dogmatische Grundentscheidungen seiner Rechtsprechung oder der konkrete Inhalt der zu prüfenden exekutiven Handlungen und Gesetze selbst. Der letzte Punkt lenkt den Blick auf eine Tatsache, der allzu selten Beachtung geschenkt wird, der Tatsache nämlich, dass es mindestens zweier Akteure bedarf, um Justizialisierung voranzutreiben: einen, der ein (potenziell verfassungswidriges) Gesetz erlässt und einen, der es wieder aufhebt. Alle vier Faktoren (Aktivierungshäufigkeit, Rollenverständnis, Dogmatik und Inhalt der geprüften Normen und Handlungen) scheinen für die Entscheidungen eines Verfassungsgerichts relevant - empirisch untersucht werden sie allerdings in den seltensten Fällen.

Demokratietheoretisch interessant ist insbesondere der Aspekt der (potenziell) verfassungswidrigen Gesetzgebungstätigkeit. Die Klage über eine überbordende Justizialisierung verkennt nämlich häufig die demokratische Funktion des judicial review: Der demokratische Rechtsstaat zeichnet sich ja nicht zuletzt dadurch aus, dass es demokratischen Mehrheiten gerade nicht erlaubt ist, jedes ihren Interessen dienende Gesetz zu implementieren, sondern legitimerweise nur solche Gesetze, die sich auch mit der übergeordneten Verfassungsordnung und den darin verankerten Grundrechten vereinbaren lassen. Nach dieser Sichtweise müssen Interventionen eines Verfassungsgerichts gegen verfassungswidrige Normen sogar als der Demokratie zuträglich verstanden werden, da durch sie eine Verletzung der Verfassung verhindert wird. Überschreitet der Gesetzgeber als „Erstinterpret“ der Verfassung seine Kompetenzen, ist es Aufgabe der Verfassungsgerichtsbarkeit, als „Zweitinterpret“ zum Schutz der Demokratie zu intervenieren (vgl. zur Unterscheidung von Erst- und Zweitinterpret Kirchhof 1998, S. 16). Justizialisierung von Politik kann in diesem Sinne als demokratische Notwendigkeit begriffen werden, deren Ausmaß von der verfassungsrechtlichen Qualität der Gesetzgebung selbst abhängig bleibt. ${ }^{2}$

Justizialisierungsprozesse - und insbesondere die passive Justizialisierung sind empirisch nur schwer zu messen. Ob Akteure tatsächlich bestimmte Hand-

2 Auch wenn man den prinzipiellen demokratietheoretischen Wert richterlicher Kontrolle anerkennt, ist damit freilich noch nicht geklärt, wann genau ein Gesetz den verfassungsrechtlichen Anforderungen nicht mehr genügt und unter welchen Umständen ein Verfassungsgericht wie tief intervenieren darf. Diese Abgrenzungsproblematik zwischen Gesetzgeber und Gericht lässt sich theoretisch auflösen, wird empirisch aber oft strittig bleiben. 
lungen wegen antizipierter Niederlagen vor Gericht oder aus völlig anderen Gründen unterlassen, ist von außen nicht ohne Weiteres erkennbar: Nicht-Handeln lässt sich in der Regel nicht messen. Allenfalls lassen sich - wie dies in den Arbeiten von Christine Landfried (s. o.) geschehen ist - über Tiefenanalysen und Einzelinterviews Erkenntnisse diesbezüglich herausarbeiten. Leichter empirisch messen lässt sich hingegen die aktive Justizialisierung. Wie häufig ein Verfassungsgericht von wem angerufen wurde und wie oft es Normen geprüft, bestätigt und verworfen hat, lässt sich ebenso an seinem Urteilsoutput ablesen wie die Antwort auf die Frage, ob bestimmte Politikbereiche stärker von Justizialisierungstendenzen betroffen sind als andere. Beide Fragekomplexe sollen im folgenden Abschnitt diskutiert und mit empirischen Daten zum Bundesverfassungsgericht unterfüttert werden. Mit ihnen kann dann auch analysiert werden, in welchem Ausmaß das Bundesverfassungsgericht tatsächlich „Vetospieler“, „Gegenregierung“ oder „Ersatzgesetzgeber" ist.

Ist es aber überhaupt sinnvoll, Verfassungsgerichte als „Vetospieler“ zu begreifen, und was heißt dies für ihre Rolle im demokratischen Prozess? Zumindest bedarf der Vetospielerbegriff einer Spezifizierung: Vetospieler im strikten Sinne George Tsebelis' (Tsebelis 1995, 2002) sind Verfassungsgerichte nicht. Sie sind es deswegen nicht, weil sie nicht per se der Veränderung eines Status quo zustimmen müssen (so aber die ursprüngliche Definition Tsebelis'). Gerichte kommen immer dann ins Spiel, wenn sie von anderen interessierten Akteuren (Bürgern, Gerichten, politischen Akteuren) eingeschaltet werden. Hat kein antragsberechtigter Akteur ein Interesse an ihrer Einschaltung, bleiben sie außen vor und treten nicht als Vetospieler in Erscheinung. Sie werden damit nicht nur , absorbiert ${ }^{6}$ (wie etwa zweite parlamentarische Kammern bei gleichgerichteten politischen Mehrheiten und Präferenzen), sondern sie werden von vornherein nicht - oder vielleicht auch erst viel später - mit einer bestimmten Frage befasst. Korrekter müsste man daher von Verfassungsgerichten als „konditionalen Vetospielern“ (Kneip 2009, S. 21) oder „triggered veto players“ (Lhotta 2003) sprechen.

Sind diese konditionalen Vetospieler aber auch „Gegenspieler“ oder „Ersatzgesetzgeber"? In beiden Formulierungen schwingen sowohl normative wie empirische Behauptungen mit, die im Folgenden einer genaueren Analyse unterzogen werden sollen. Beide Begriffe lassen sich im Grunde auf zwei Fragen beziehen: Wie häufig greifen Verfassungsgerichte an Stelle der Parlamente und Regierungen in politische Prozesse ein, wie häufig „kassieren“ sie demokratisch legitimierte Vorhaben des Gesetzgebers? Und urteilen sie dabei, wie es der Begriff des Gegenspielers vermuten lässt, häufig entgegen der inhaltlichen Intentionen der jeweiligen politisch Verantwortlichen? Beide Fragen sollen, zusammen mit der Justizialisierungsthese, anhand des Urteilsoutputs des Bundesverfassungsgerichts diskutiert werden.

\section{Justizialisierungseffekte durch verfassungsgerichtliches Agieren 1951-2005}

Ob das Bundesverfassungsgericht in der Vergangenheit tatsächlich ,Gegen-' oder ,Nebenregierung' der gewählten Exekutiven und Legislativen gewesen ist und aktiv zur Justizialisierung der Politik beigetragen hat, lässt sich am prägnantesten 
anhand jener Verfahren untersuchen, bei denen die Prüfung von Normen eine Rolle spielte. ${ }^{3}$ Normenkontrolle findet nicht nur in den entsprechenden abstrakten und konkreten Normenkontrollverfahren statt, sondern im bundesdeutschen Fall in überwiegendem Maße im Rahmen von Verfassungsbeschwerden. Es reicht daher nicht aus, sich nur auf die klassischen Normenkontrollverfahren zu kaprizieren, sondern es ist nötig, alle drei Verfahrenarten in die Analyse einzubeziehen.

Hinsichtlich der Justizialisierungseffekte drängen sich zunächst drei Fragen auf: Erstens muss danach gefragt werden, wie häufig das Gericht überhaupt in die demokratische Gesetzgebung eingreift, indem es Normen prüft und annulliert. Zweitens ist zu fragen, ob die Prüfintensität von Normen über die Zeit zugenommen hat - ob das Bundesverfassungsgericht also nicht nur häufiger Normen kontrolliert, sondern auch häufiger annulliert hat. Die implizite Annahme der Justizialisierungsthese scheint zu sein, dass der Einfluss der Verfassungsgerichtsbarkeit über die Zeit zugenommen hat. Ob diese Annahme tatsächlich zutrifft, lohnt daher eine Überprüfung. Drittens schließlich kann analysiert werden, ob Varianzen bezüglich unterschiedlicher Politikfelder zu beobachten sind - ob also bestimmte Politikbereiche häufiger als andere Gegenstand verfassungsgerichtlicher Überprüfung gewesen sind.

\subsection{Justizialisierung im Zeitverlauf}

Die grundlegenden Daten für den hier interessierenden Analysezeitraum 19512005 finden sich bereits in der Jahresstatistik des Bundesverfassungsgerichts. Ausweislich der Jahresstatistik 2005 hat das Gericht zwischen 1951 und 2005 insgesamt 45 Bundes- und 19 Landesnormen (Gesetze und Verordnungen) sowie 372 Einzelnormen des Bundes und 145 Einzelnormen der Länder ganz oder teilweise als verfassungswidrig beanstandet (http://www.bverfg.de/organisation/gb2005/AVI.html). Was zunächst nach einer erheblichen Anzahl klingt, erweist sich bei genauerer Betrachtung als nicht sehr hoch, da das Gericht im gleichen Zeitraum insgesamt etwa 155.000 Verfahren entschieden hat, darunter 3.361 abstrakte und konkrete Normenkontrollverfahren sowie knapp 150.000 Verfassungsbeschwerden. Bezogen auf die insgesamt entschiedenen 154.502 Verfahren in diesem Zeitraum nehmen sich die beanstandeten 581 Gesetze und Einzelnormen des Bundes und der Länder also vergleichsweise gering aus. Statistisch wurde damit nur in etwa 0,4 Prozent aller entschiedenen Fälle eine (einzelne) Bundes- oder Landesnorm moniert.

Allerdings verbergen sich hinter den etwa 155.000 Verfahren auch solche, in denen überhaupt keine Normprüfung in der Sache stattgefunden hat, weil die Klagen entweder gleich aus formalen Gründen zurückgewiesen wurden oder weil es sich um Verfahren handelte, die schon ihrer Natur nach keine Normprüfung

3 Auch kompetenzrechtliche Verfahren (also vor allem das Organstreitverfahren) können im Ergebnis ein aktives ,Hineinregieren' in den politischen Prozess bedeuten. Die Beilegung von Kompetenzstreitigkeiten durch Verfassungsgerichte ist in der Regel aber nicht umstritten und die Notwendigkeit ihrer Schiedsrichterfunktion weithin anerkannt. Im Normenkontrollverfahren hingegen zeigen sich nicht nur Aktivismus und Zurückhaltung eines Verfassungsgerichts in besonderer Weise, auch die demokratietheoretische Relevanz des ,Hineinregierens' ist ungleich größer. 
zum Gegenstand hatten (also etwa reine Urteilsverfassungsbeschwerden, Organstreitverfahren, Parteiverbotsverfahren, etc.). Auch lässt sich aus diesen Zahlen keine Entwicklung der Normenkontrollaktivitäten des Bundesverfassungsgerichts über die Zeit herauslesen. Notwendig ist daher eine genauere Analyse des verfassungsgerichtlichen Urteilsoutputs.

Abbildung 1 zeigt die Anzahl annullierter Bundesgesetze für die Jahre 1951 bis $2005 .{ }^{4}$ Was sogleich auffällt: Die Anzahl der durch das Bundesverfassungsgericht seit Aufnahme seiner Tätigkeit annullierten Bundesnormen hat sich im Zeitverlauf nicht generell erhöht. Die Vermutung, dass das Gericht den Spielraum des Gesetzgebers im Laufe der Zeit immer weiter eingeschränkt und immer häufiger Normen annulliert haben könnte, wird durch die empirische Datenlage nicht gestützt und kann insoweit zurückgewiesen werden. Im Gegenteil: Obwohl die Gesamtzahl der vor dem Bundesverfassungsgericht verhandelten Verfahren seit den 1950er-Jahren nahezu stetig gewachsen ist (von ca. 500 Verfahrenseingängen im Jahr 1951 auf mehr als 5.000 im Jahr 2005; vgl. Kneip 2009, S. 208), ist kein absoluter Anstieg annullierter Normen zu verzeichnen. Vielmehr schwankt die Anzahl der in den verschiedenen Jahren annullierten Bundesnormen zwischen keiner Annullierung (in den Jahren 1952, 1954 und 1997) und der Annullierung von 14 Normen im Jahr 1998. Eher lassen sich ,Annullierungswellen' zwischen 1955 und 1973 (mit Scheitelpunkten 1964 und 1971) und zwischen 1974 und 1996 (mit einem Scheitelpunkt 1986), sowie eine deutlich erhöhte Annullierungshäufigkeit zwischen 1998 und 2005 (mit Ausnahme allerdings der Jahre 2001 und 2002, die nur eine unterdurchschnittliche Annullierungshäufigkeit aufweisen) feststellen.

4 Abbildung 1 zeigt - wie auch die folgenden Abbildungen - die Anzahl annullierter (bzw. geprüfter) Normen nach dem Jahr der Entscheidung des Bundesverfassungsgerichts. Damit werden weder Aussagen über die jeweilige Verfahrensdauer noch über das Antragsverhalten unterschiedlicher Akteure im Zeitverlauf getroffen; dargestellt sind ausschließlich Veränderungen im Annullierungsverhalten des Gerichts selbst. Zwischen Antragstellung und endgültiger Entscheidung des Gerichts können leicht mehrere Jahre vergehen. 


\section{Abbildung 1: Nichtige und/oder mit dem Grundgesetz unvereinbare Bundesnormen (1951-2005)}

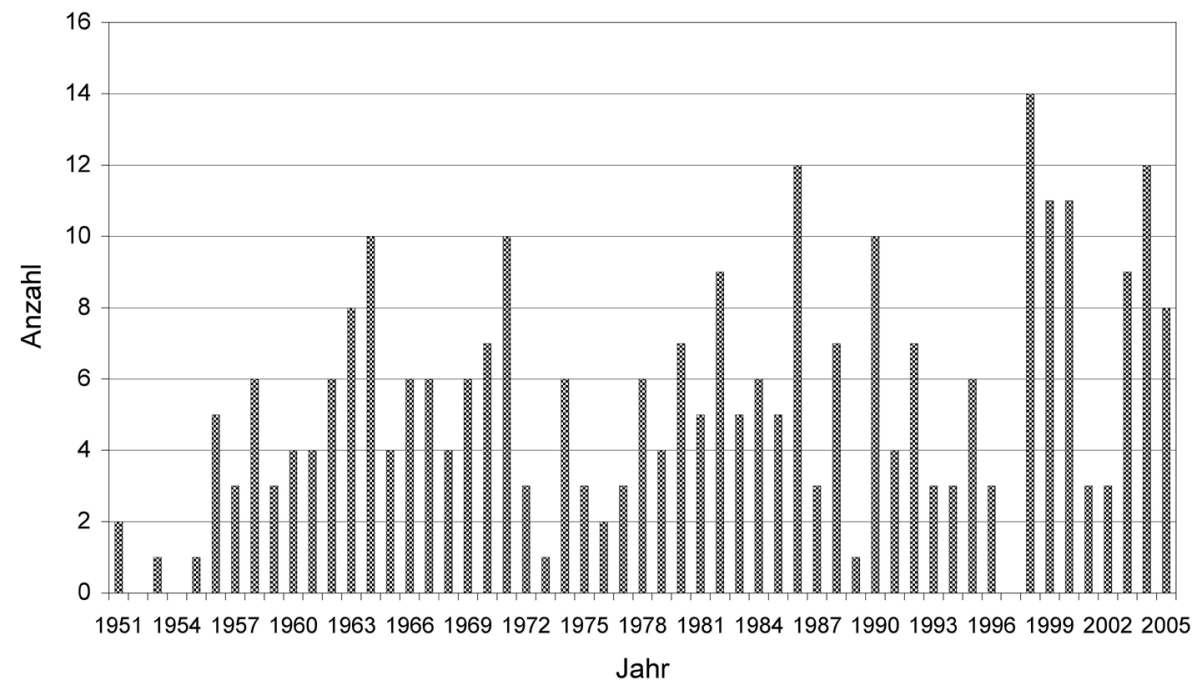

Quellen: Datenhandbuch zur Geschichte des deutschen Bundestages 1949-2003; eigene Auszählung auf Basis der amtlichen Entscheidungssammlung des Bundesverfassungsgerichts.

Damit kann schon hier die These zurückgewiesen werden, dass das Regierungssystem der Bundesrepublik Deutschland prinzipiell zunehmenden Justizialisierungstendenzen ausgesetzt sei. Zwar wird das Bundesverfassungsgericht immer häufiger angerufen; versteht man unter „Justizialisierung der Politik“ aber nicht nur ein häufigeres Aktivieren, sondern auch ein zunehmendes Hineinregieren des Bundesverfassungsgerichts in die Politik, so verdeutlicht Abbildung 1, dass dies für die Bundesrepublik zumindest nicht generell zutrifft: Das Gericht hat im Zeitverlauf keineswegs immer häufiger (Bundes-)Normen annulliert, sondern die Annullierungshäufigkeiten unterliegen offenkundig bestimmten Konjunkturen. ${ }^{5}$

Die absolute Anzahl beanstandeter Bundesnormen gibt die tatsächliche Prüftätigkeit des Bundesverfassungsgerichts aber ohnehin nur unvollständig wieder. Ein vollständiges Bild des Annullierungsverhaltens des Bundesverfassungsgerichts er-

5 Nicht erfasst werden kann mit dieser Unterscheidung die Tatsache, dass das Bundesverfassungsgericht auch bei Normbestätigung mitunter durch verbindliche Interpretationen des Normtextes faktisch in den Aufgabenbereich des Gesetzgebers hineinregiert (ich danke den beiden anonymen Gutachterinnen und Gutachtern für diesen wichtigen und richtigen Punkt). Insbesondere das Instrument der „verfassungskonformen Auslegung“ von Normen kann den Spielraum der Politik potentiell erheblich begrenzen, ohne dass eine Norm formal aufgehoben oder für unvereinbar mit der Verfassung erklärt werden müsste (siehe für einige prägnante Beispiele z. B. Schlaich u. Korioth 2004, S. 314-315 sowie für eine kritische Bestandsaufnahme Zippelius 1976 und Voßkuhle 2000). Gleichwohl ist die verfassungskonforme Auslegung von ihrem Prinzip her als selbstbeschränkendes Instrument gedacht, das die Einheit der Rechtsordnung sichern und gerade den Vorrang des Gesetzgebers bei der Konkretisierung der Verfassung zum Ausdruck bringen soll. Insbesondere die gesetzgeberischen Grundentscheidungen, Wertungen und Zwecke einer Norm dürfen durch verfassungskonforme Auslegung prinzipiell nicht verändert werden (siehe z. B. klassisch Hesse 1999 [1966], 
gibt sich erst, wenn die beanstandeten Normen zur Gesamtzahl verfassungsgerichtlicher Normprüfungen in Beziehung gesetzt werden. Schlüsselt man diese nach den Verfahrensarten Verfassungsbeschwerde und konkrete Normenkontrol$1 e^{6}$ auf, ergibt sich folgendes Bild: Bezüglich der Verfassungsbeschwerdeverfahren finden sich in der amtlichen Entscheidungssammlung des Bundesverfassungsgerichts zwischen den Jahren 1951 und 2005 insgesamt 641 Verfassungsbeschwerdeverfahren, in denen Gesetzesnormen des Bundes oder der Länder direkt oder indirekt Prüfgegenstand des Gerichts waren. Betrachtet man den prozentualen Anteil der annullierten Normen an allen in einem Jahr entschiedenen Verfassungsbeschwerdeverfahren mit Normprüfung, bestätigt sich die oben schon für die Bundesnormen getroffene Aussage, dass über die Zeit keine generelle Zunahme der Annullierungshäufigkeit von Gesetzen festgestellt werden kann (vgl. Abb. 2). Nach anfänglich kaum vorhandener Annullierungstätigkeit stabilisiert sich die Annullierungsquote Ende der 1950er-Jahre bis zu Beginn der 1980er-Jahre auf moderatem Niveau, bevor seit Mitte der 1980er-Jahre starke Ausschläge nach oben und unten in unterschiedlichen Jahren zu beobachten sind, ohne dass eine generelle Zunahme der Annullierungstätigkeit konstatiert werden müsste. Mit dem Anstieg und dem Rückgang von Bestätigungen steigt und sinkt meist auch die Annullierung von Normen, wobei insgesamt weit mehr Normen bestätigt als annulliert wurden. Auch dies spricht nicht dafür, dem Gericht eine prinzipielle Gegenspielerrolle zur Politik allgemein oder gar einer bestimmten Regierungskonstellation gegenüber zu unterstellen. Auffällig ist aber, dass seit den späten 1990erJahren mehr Normen pro Jahr annulliert als bestätigt wurden, was möglicherweise doch auf eine zunehmende Justizialisierung seit Mitte der 1990er Jahre hindeutet. Zwischen 1995 und 2005 überstieg die Anzahl der Annullierungen jene der Bestätigungen in immerhin 7 von 11 Jahren; das Verhältnis von Bestätigung und Annullierung hat sich seit Ende der 1990er-Jahre also tendenziell umgekehrt.

S. 30 ff., die Ausführungen des BVerfG zu den Grenzen der verfassungskonformen Auslegung in BVerfGE 54, 277, S. 299 sowie Schlaich u. Korioth 2004, S. 313-314). Das Instrument selbst ist daher als ein grundsätzlich nicht-interventionistisches konzipiert. Im Rahmen dieser Untersuchung wird unterstellt, dass die Anwendung der verfassungskonformen Auslegung in der Regel tatsächlich eher der Anerkennung des Vorrangs des Gesetzgebers bei der Konkretisierung der Verfassung dient und weniger einer Intervention , durch die Hintertür ${ }^{6}$ - wenngleich nicht ausgeschlossen werden soll, dass dem in der faktischen Wirkung mitunter doch so ist. Quantitativ erfassen lässt sich die Wirkung verfassungskonformer Auslegungen letztlich nicht.

6 Verfassungsbeschwerden und konkrete Normenkontrollen machen etwa 98 Prozent aller vom Bundesverfassungsgericht entschiedenen Verfahren aus. Auf einen Einbezug der abstrakten Normenkontrolle soll daher hier verzichtet werden. Für diese ist ohnehin seit Langem bekannt, dass hier die Annullierungswahrscheinlichkeit für eine geprüfte Norm bei etwa 50 Prozent liegt (vgl. Stüwe 1997, 2001; Kneip 2009). 
Abbildung 2: Prozentualer Annullierungsanteil von Normen (Landes- und Bundesnormen) im Verfassungsbeschwerdeverfahren (1951-2005)

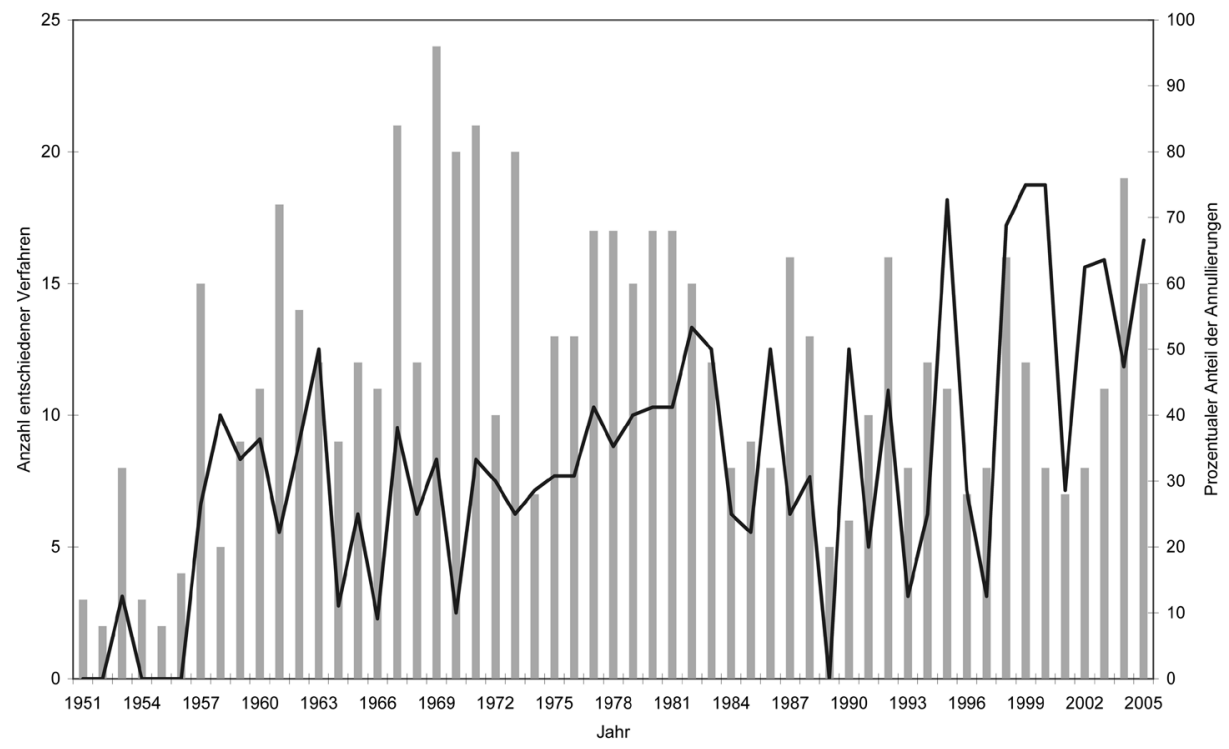

Quelle: Eigene Darstellung auf Basis der Auswertung von 641 Verfassungsbeschwerdeverfahren der amtlichen Entscheidungssammlung des Bundesverfassungsgerichts. Die grau hinterlegten Balken bilden die Anzahl aller in einem Jahr entschiedenen Verfassungsbeschwerdeverfahren mit Normprüfung $a b$.

Ein ähnliches Bild ergibt sich für das konkrete Normenkontrollverfahren. Bis Ende der 1980er-Jahre hat das Bundesverfassungsgericht auch über diesen Verfahrensweg pro Jahr deutlich mehr Normen bestätigt als annulliert, und die Annullierungsanteile sind in diesem Zeitraum sogar leicht rückläufig. Erst seit den 1990er-Jahren steigen die Annullierungsquoten - bei allerdings deutlich rückläufigen Verfahrenszahlen - wieder an, aber auch hier sind deutliche Varianzen über die Jahre hinweg feststellbar (vgl. Abbildung 3). 


\section{Abbildung 3: Prozentualer Annullierungsanteil von Normen (Landes- und Bundesnormen) im konkreten Normenkontrollverfahren (1951-2005)}

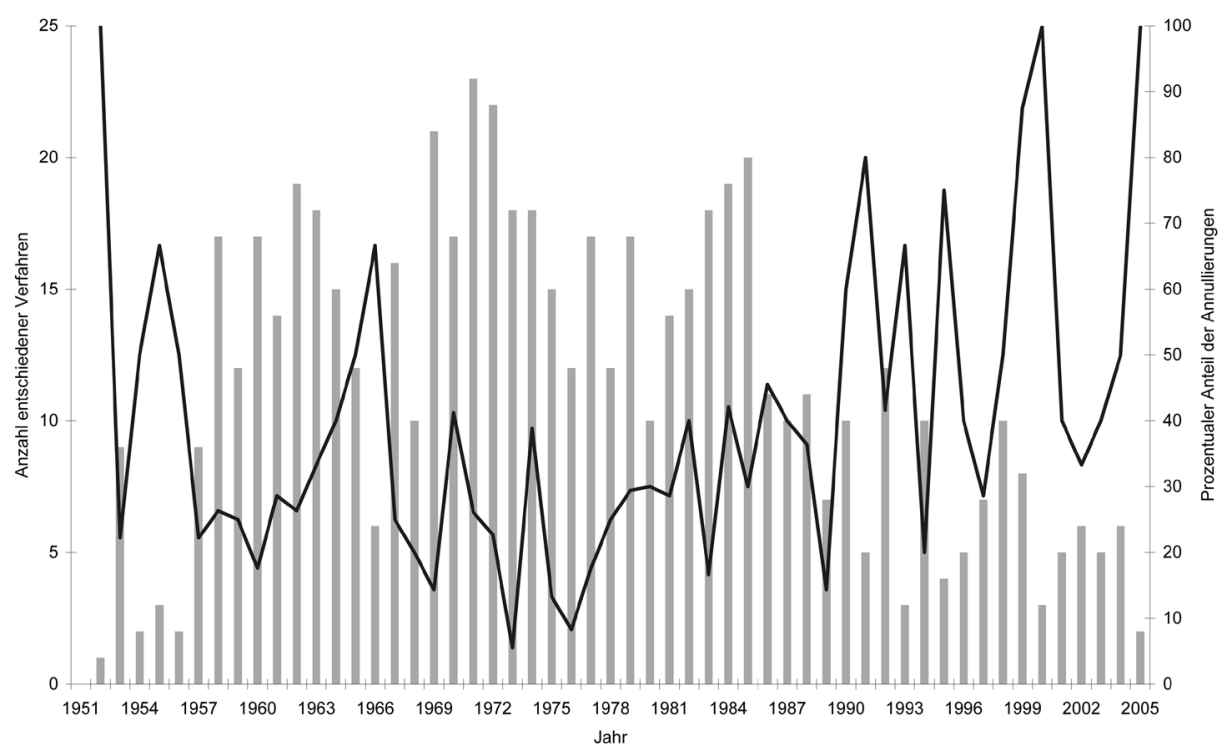

Quelle: Eigene Darstellung auf Basis der Auswertung von 590 konkreten Normenkontrollverfahren der amtlichen Entscheidungssammlung des Bundesverfassungsgerichts. Die grau hinterlegten Balken bilden die Anzahl aller in einem Jahr entschiedenen konkreten Normenkontrollverfahren ab.

Die insgesamt überprüften 1231 Verfassungsbeschwerde- und konkreten Normenkontrollverfahren lassen also nicht den generellen Schluss zu, dass das Bundesverfassungsgericht schon über die bloße Anzahl der entschiedenen Normenkontrollverfahren übermäßig stark auf die Politik Einfluss genommen hätte. Allenfalls für die letzten zehn Jahre des Untersuchungszeitraums kann eine leicht zunehmende Justizialisierungstendenz bei gleichzeitig deutlich zunehmender Varianz festgestellt werden. ${ }^{7}$

Da die absolute Zunahme von Verfahren offenbar keine zufriedenstellende Erklärung für diesen Trend liefert, bieten sich zwei andere Erklärungen an: Zum einen könnte das Gericht seine eigene Rollenwahrnehmung im demokratischen Prozess verändert haben und schlicht, annullierungsfreudiger' geworden sein entweder, weil es seine Rolle nun ,aktiver` definiert, oder weil sich seine Recht-

7 Insgesamt liegt die Bestätigungsquote von Normen über alle Verfahren hinweg bei etwa 2/3, d. h., ca. 66 Prozent aller Verfahren im Untersuchungszeitraum enden mit einer Bestätigung der überprüften Norm, etwa 34 Prozent mit einer Annullierung. Kleinere Unterschiede zeigen sich hinsichtlich der Verfahrensarten und der betroffenen Politikbereiche (vgl. Kneip 2009, S. 293-308). Hinsichtlich des vergleichsweise hoch anmutenden Anteils von Annullierungen sei nochmals daran erinnert, dass es sich bei den hier ausgewerteten Verfahren nur um die in der amtlichen Entscheidungssammlung des Gerichts veröffentlichten Senatsentscheidungen (und nicht alle Entscheidungen des Gerichts) handelt und auch kleinere Teilnichtigkeits- oder Unvereinbarkeitserklärungen in die Zählung eingeflossen sind (siehe hierzu auch die Ausführungen weiter oben unter 3.1). 
sprechungsdogmatik gewandelt hat. Die leicht zunehmende Justizialisierung wäre also einem zunehmenden Aktivismus des Gerichts selbst geschuldet. Zum anderen könnte die zunehmende Annullierungshäufigkeit auf eine Zunahme verabschiedeter verfassungswidriger Gesetze zurückzuführen sein. In diesem Fall wäre die Justizialisierung eine Folge handwerklich ,schlechter' Gesetzgebung. Für beide Annahmen gibt es Fürsprecher in der Literatur (z. B. Schneider 1999 für die erste, Benda 1979 für die zweite Auffassung), beide Annahmen bedürfen aber der empirischen Überprüfung. Ein erster Schritt in diese Richtung kann getan werden, wenn die Annullierungshäufigkeit nach Politikfeldern unterschieden und damit die Möglichkeit eröffnet wird, die spezifischen Fälle, die hinter den Annullierungen stehen, genauer zu betrachten.

\subsection{Justizialisierung nach Politikfeldern}

Die drei am häufigsten von verfassungsgerichtlichen Eingriffen betroffenen Politikfelder sind traditionell die Rechtspolitik, die Sozialpolitik und die Steuer- und Finanzpolitik (vgl. z. B. Benda 1979; Beyme 1985, 1987; Landfried 1994; Kneip 2006, 2009). In diesen drei Politikfeldern werden etwa dreimal so viele Normen geprüft und annulliert wie in allen anderen Politikfeldern zusammen. Abbildung 4 zeigt die Annullierungssaldi ${ }^{8}$ der drei relevanten Politikfelder im Zeitverlauf. Auch hier lässt sich das oben bereits diskutierte Muster erkennen: Im Großen und Ganzen bewegt sich der Annullierungssaldo - mit Ausnahme einzelner Jahre in den Politikfeldern Sozialpolitik und Steuer- und Finanzpolitik - über einen langen Zeitraum hinweg im positiven Bereich, bevor sich diese Tendenz mit Beginn der 1990er-Jahre zu verändern beginnt, ohne jedoch vollends in den negativen Skalenbereich zu fallen. Die Gründe für diese Entwicklung sind in jedem der drei Politikfelder unterschiedlich, wie eine genauere Analyse der hinter den Zahlen stehenden Verfahren zeigt.

8 Der Annullierungssaldo bildet das Verhältnis von Normbestätigungen zu Normannullierungen für die betrachteten Politikfelder und in der Summe ab. Werden mehr Normen pro Jahr bestätigt als annulliert, ist der Annullierungssaldo positiv, werden mehr Normen annulliert als bestätigt, ist er negativ. 


\section{Abbildung 4: Annullierungssaldi im Zeitverlauf}

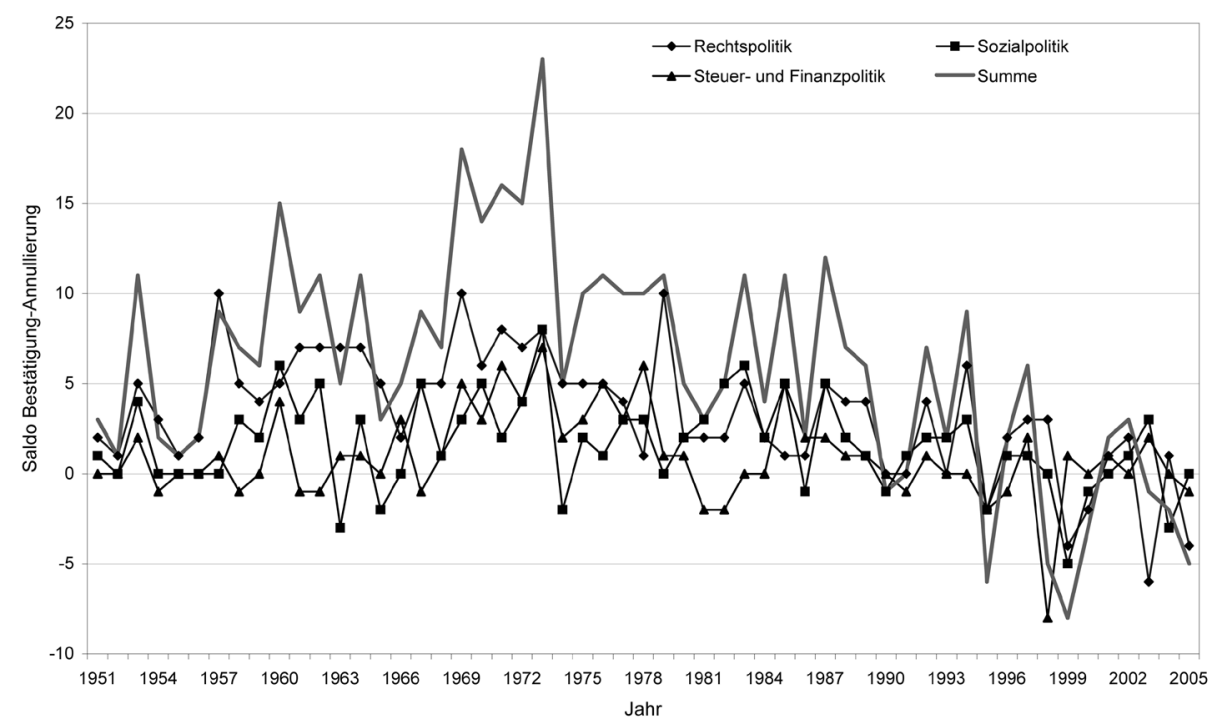

Quelle: eigene Darstellung.

Für den Bereich der Steuer- und Finanzpolitik fallen zwei Dinge auf: Eine deutliche generelle Zunahme von Verfahren in diesem Politikfeld in den 1970er-Jahren (vgl. Kneip 2009, S. 302-303) und eine außergewöhnlich hohe Annullierungsquote im Jahr 1998. Die generelle Verfahrenszunahme scheint mit einer erhöhten gesetzgeberischen Tätigkeit in diesem Politikfeld erklärt werden zu können. Nach ersten größeren Steuer- und Finanzreformen Mitte der 1950er-Jahre (vgl. Schmidt 2001) fand die Reformtätigkeit ihren ersten Höhepunkt im Finanzreformgesetz von 1969 und in der grundlegenden Reform der Einkommensteuer und der Abgabenordnung in den Jahren 1974/75, durch welche die seit 1925 bzw. 1919 geltenden steuerrechtlichen Grundlagen fundamental reformiert wurden (vgl. Jäger 1986, S. 139-140; siehe auch Wagschal 2006). Folge des gesetzgeberischen Handelns war es, dass alleine das Einkommensteuergesetz zwischen 1969 und 1979 $17 \mathrm{Mal}$ zum Gegenstand verfassungsgerichtlicher Überprüfung wurde. Dreimal wurden zwischen 1973 und 1976 zudem verschiedene Normen des Umsatzsteuergesetzes einer Überprüfung unterzogen. Kurzum: Die große Anzahl der Verfahren im Politikfeld Steuer- und Finanzpolitik lässt sich zu einem großen Teil auf eine erhöhte gesetzgeberische Tätigkeit und anschließende Klagen gegen die Veränderungen des Status quo zurückführen. Trotz dieser großen Zunahme ist aber keine Erhöhung des Annullierungssaldos zu erkennen. Im Gegenteil - er liegt in den 1970er-Jahren deutlich stärker im positiven Bereich als zuvor und danach. Mit anderen Worten: Ein direkter Zusammenhang zwischen der Anzahl der an das Bundesverfassungsgericht herangetragenen Verfahren und der Annullierungsquote ist in diesem Politikfeld nicht erkennbar. 
Sehr gut erkennbar ist hingegen die hohe Annullierungsquote des Jahres 1998. Sie ist im Kern durch eine Rechtsprechungsänderung des Bundesverfassungsgerichts selbst zu erklären. Unter Federführung des zuständigen Berichterstatters Paul Kirchhof hatte das Gericht Mitte der 1990er-Jahre begonnen, seine Rechtsprechung zum Familien- und Kinderexistenzminimum zu präzisieren und weiterzuentwickeln (vgl. auch Köppe 2006). Gleich fünf Annullierungen aus dem Jahr 1998 betrafen das Einkommensteuergesetz, und auch das im gleichen Jahr beanstandete Steuersenkungsgesetz verstieß im Kern gegen die von Karlsruhe neu aufgestellten Maßstäbe zum Existenzminimum. In gleich vier am selben Tag veröffentlichten Urteilen erklärte das Gericht die „gesamte Familienpolitik früherer Bundesregierungen " für verfassungswidrig (Schneider 1999, S. 1303). Die hohe Anzahl von Annullierungen im Jahr 1998 ist damit nicht auf eine erhöhte Tätigkeit des Gesetzgebers oder eine allgemeine Zunahme von Klagen in diesem Politikbereich zurückzuführen, sondern auf eine veränderte Rechtsprechung des Gerichts selbst (vgl. insb. BVerfGE 99, 216; 99, 246; 99, 268; 99, 273).

Ein etwas anderes Bild ergibt sich, wenn man die bestätigten und annullierten Normen im Politikfeld Sozialpolitik betrachtet. Wie in der Steuer- und Finanzpolitik wurden auch in der Sozialpolitik in den meisten Jahren wesentlich mehr Normen bestätigt als annulliert, mit Ausreißern vor allem in den Jahren 1963 und 1999. Und auch hier zeigt sich eine leichte Zunahme der Annullierungen in der zweiten Hälfte der 1990er-Jahre, deren Gründe diesmal allerdings nicht in einer Veränderung der Rechtsprechung des Gerichts, sondern in einer erhöhten Reformtätigkeit des Gesetzgebers angesichts historischer Umbruchsituationen zu finden sind. Im Jahr 1963 hatte Karlsruhe vor allem über die sozialpolitische Flankierung der Kriegsfolgen zu befinden. Gegenstand der in diesem Jahr zu verzeichnenden Annullierungen waren in erster Linie das Kriegsgefangenenentschädigungsgesetz, die gesetzlichen Regelungen der Versorgungsansprüche ehemaliger NS-Beamter und das Bundesversorgungsgesetz, das 1957 grundlegend reformiert worden war. In allen Fällen mühte sich der Gesetzgeber (vergeblich) um eine verfassungskonforme Regelung sozialpolitischer Ansprüche.

Um solche ging es auch bei vier der sechs Annullierungen des Jahres 1999. Mit dem Anwartschaftsüberführungsgesetz und dem Rentenüberleitungsgesetz hatte der Gesetzgeber versucht, die Rentenanwartschaften ehemaliger DDR-Bürger zu regeln. Auch hier hielten die Karlsruher Richterinnen und Richter die gefundenen Lösungen für nicht mit dem Grundgesetz vereinbar. Beide ,Annullierungsausreißer' sind also, wenn man so will, mit zwei historischen Sondersituationen und den sich daran anschließenden politisch-rechtlichen Regelungsversuchen zu erklären: der Neugestaltung der Rechtsverhältnisse nach Ende des Nationalsozialismus und der deutsch-deutschen Wiedervereinigung samt der entsprechenden sozialrechtlichen Detailregelungen. ${ }^{9}$ Die Zunahme der Annullierungsquote lässt sich

9 Die Überleitung sozialpolitischer Rechte und Ansprüche spielte auch in den Jahren vor und nach 1963 respektive 1999 eine nicht unerhebliche Rolle für die Arbeit des Bundesverfassungsgerichts, wenngleich nicht so auffällig wie in diesen beiden Jahren. Sowohl während der 1960er-Jahre als auch in den Jahren nach 1990 hatte das Gericht immer wieder Entscheidungen zu diesen Fragen zu treffen. 
weder auf generell gestiegene Verfahrenszahlen noch auf eine veränderte Rechtsprechung des Bundesverfassungsgerichts zurückführen.

Für das dritte hier näher zu untersuchende Politikfeld der Rechtspolitik ist auffällig, dass im Gegensatz zu den beiden zuvor betrachteten Politikfeldern der Anteil der Gesetzesannullierungen erst gegen Ende der 1990er-Jahre deutlicher zulegt. Während in der Steuer- und Finanzpolitik und der Sozialpolitik immer mal wieder in einzelnen Jahren negative Annullierungssaldi sichtbar werden, lässt sich dies für die Rechtspolitik erst Mitte der 1990er-Jahre beobachten. Die Gründe hierfür scheinen aber weder in historischen Sondersituationen oder besonders umstrittenen (und dadurch häufig geprüften) einzelnen Gesetzen zu liegen. Eher scheint die Erklärung für zunehmende Annullierungen im Zusammenspiel einer (beibehaltenen) liberalen Rechtsprechung des Gerichts einerseits und einer zunehmenden Verabschiedung rechtsstaatsbeschränkender Gesetze andererseits zu liegen. Diese Vermutung drängt sich zumindest bei Durchsicht der geprüften und annullierten Normen seit Mitte der 1990er-Jahre auf: Zu den seitdem geprüften (und ganz oder teilweise annullierten) Normen gehören beispielsweise das so genannte „Verbrechensbekämpfungsgesetz“ (BVerfGE 100, 313), das Gesetz über den „Großen Lauschangriff“ (BVerfGE 109, 279), das Gesetz zum Europäischen Haftbefehl (BVerfGE 113, 273), das niedersächsische „Gesetz über die öffentliche Sicherheit und Ordnung " (BVerfGE 113, 348), das Luftsicherheitsgesetz (BVerfGE 115, 118), die Gesetze zur automatischen Kennzeichenerfassung von Hessen und Schleswig-Holstein (BVerfGE 120, 378), das nordrhein-westfälische Verfassungsschutzgesetz („Online-Durchsuchung“; BVerfGE 120, 274), das Telekommunikationsgesetz („Vorratsdatenspeicherung“, einstweilige Anordnungen z.B. BVerfGE 121, 1; 121, 391; 122, 120; 123, 89; 124, 299) sowie das bayerische Versammlungsgesetz (einstweilige Anordnung; BVerfGE 122, 342). In all diesen Fällen beanstandete das Bundesverfassungsgericht - unter Beibehaltung der großen Linien seiner Rechtsprechung - freiheitsbeschränkende Gesetze des Bundes und der Länder.

Zugleich schärfte Karlsruhe, auch unter Einfluss der Rechtsprechung des Europäischen Gerichtshofs für Menschenrechte ${ }^{10}$, seine Rechtsprechung im Bereich des Kindschafts- und Familienrechts (sowie des Ausländerrechts, sofern Rechte ausländischer Familienmitglieder in den jeweiligen familienrechtlichen Regelungen nicht ausreichend berücksichtigt waren; siehe beispielsweise das Urteil gegen das Ausländergesetz aus dem Jahr 2005 [BVerfGE 114, 357]). Beide Rechtsprechungsgegenstände - liberale Abwehrrechte und Kindschafts- und Familienrecht - machen einen Großteil der nach 1990 annullierten Normen im Politikfeld Rechtspolitik aus.

10 Der Einfluss der europäischen Gerichtsbarkeit (EuGH und EMRGH) auf die Justizialisierung in der Bundesrepublik müsste einer gesonderten Betrachtung unterzogen werden, wie ohnehin die Konsequenzen der europäischen Mehrebenenordnung auf nationale Rechtssysteme einer stärkeren Beachtung bedürften. Für den EMRGH sei an dieser Stelle nur erwähnt, dass nicht wenige Karlsruher Interventionen der jüngsten Zeit in den Bereichen Familienrecht, Persönlichkeitsrecht („Caroline-Urteile“) und Strafrecht („Sicherungsverwahrung“) ihren Ausgangspunkt in Straßburg hatten bzw. dort angestoßen wurden (siehe zum Verhältnis der beiden Gerichte Mückl 2005). 
Die gestiegenen Annullierungsquoten seit den 1990er-Jahren in den drei untersuchten Politikfeldern sind also unterschiedlichen Ursachen geschuldet. Im Bereich der Steuer- und Finanzpolitik liegen sie vor allem in einer veränderten Rechtsprechung des Gerichts zum Existenzminimum, im Bereich der Sozialpolitik zu einem Großteil an der historisch bedingten Sondersituation der Überleitung des DDR-Sozialsystems in das bundesdeutsche und im Bereich der Rechtspolitik an einer tendenziell rechtsstaatseinschränkenden Gesetzgebung in Bund und Ländern.

Zwei allgemeine Ergebnisse dieses Abschnitts sollten mit Blick auf die Justizialisierungsthese nochmals festgehalten werden: Erstens ist gezeigt worden, dass nicht alleine anhand der absoluten Anzahl annullierter Normen auf eine aktivistische oder zurückhaltende Normenkontrolltätigkeit des Bundesverfassungsgerichts rückgeschlossen werden kann. Um beurteilen zu können, wie stark das Gericht tatsächlich ,in die Politik hineinregiert', müssen vielmehr auch die bestätigten Normen in die Betrachtung miteinbezogen werden. Hier zeigt sich, dass das Bundesverfassungsgericht in der Vergangenheit weit mehr Normen bestätigt als annulliert hat, also im Großen und Ganzen - gemessen an der Vielzahl der Verfahren - nicht übermäßig häufig in die politische Sphäre ,hineinregiert ${ }^{`}$ hat. Allerdings hat die Annullierungsquote in den 1990er-Jahren merklich zugenommen, insbesondere im Verfassungsbeschwerdeverfahren.

Zweitens ist gezeigt worden, dass sich die Annullierungshäufigkeit nach Politikfeldern unterscheidet. Obwohl absolut am häufigsten von Annullierungen betroffen, ist im Politikfeld Rechtspolitik über den gesamten Untersuchungszeitraum hinweg die geringste Annullierungsquote zu beobachten, gefolgt von der Sozialpolitik und der Steuer- und Finanzpolitik. Alle drei Politikbereiche weisen aber einen deutlich positiven Annullierungssaldo auf; erst in der zweiten Hälfte der 1990er-Jahre verlagert er sich leicht in den negativen Bereich - erst seitdem werden also tendenziell mehr Normen annulliert als bestätigt.

Die Analyse der faktischen Normenkontrolltätigkeit des Gerichts lässt daher insgesamt nicht den Schluss zu, dass die Karlsruher Richter aktivistisch in die Politik hineinregieren. Das Gericht scheint über die Zeit hinweg, ungeachtet seiner personellen Zusammensetzung und der politischen Mehrheiten, denen es sich gegenübersah, eher gleichmäßig zwischen Aktivismus und Zurückhaltung gewählt zu haben - mit deutlicher Tendenz zur Zurückhaltung. Ein vom Bundesverfassungsgericht ausgehender Justizialisierungstrend lässt sich damit nicht feststellen. Allerdings kann auch nicht übersehen werden, dass die Annullierungshäufigkeit gegen Ende des Untersuchungszeitraums merklich zugenommen hat, und dass in immerhin fast jedem dritten in der Sache entschiedenen Fall eine Landes- oder Bundesnorm durch das höchste deutsche Gericht beanstandet wurde. Ist das Gericht damit also doch gewichtiger Gegenspieler zu Parlament und Regierung in der bundesdeutschen Politik? Mischt es sich doch häufiger in die Politik ein als es sollte? Diese Frage lässt sich nicht anhand der bloßen Verfahrenszahlen beantworten. Vielmehr müssen die Zahlen in Bezug zur Gesetzgebung und zur Funktion und Aufgabe des Bundesverfassungsgerichts in der bundesdeutschen Demokratie gesetzt werden. Dies soll im nächsten Abschnitt geschehen. 


\section{Demokratiefunktionales Agieren des Bundesverfassungsgerichts}

Bedenkt man die Häufigkeit der Befassung des Bundesverfassungsgerichts mit Klagen von politischen Akteuren und Bürgern oder Vorlagen durch Gerichte, dann ist Karlsruhe in den letzten sechzig Jahren, wie gesehen, nicht übermäßig häufig als Vetospieler in Erscheinung getreten. Nun ist mit den Begriffen „Gegenspieler" oder „Gegenregierung“ aber doch mehr gemeint als nur ein numerisch häufiges Eingreifen - in ihnen schwingt ein Unbehagen darüber mit, dass das Bundesverfassungsgericht inhaltlich Dinge entscheidet, die vermeintlich nicht von ihm, sondern von gewählten Legislativen und Exekutiven entschieden werden sollten. Dieses Unbehagen ist grundsätzlich nachvollziehbar, beruht Demokratie doch idealtypisch auf der Idee der Volkssouveränität (siehe grundlegend Maus 1991; Abromeit 1999) und nicht auf einer wie immer gearteten ,Richtersouveränität ${ }^{`}$. Es ist aber insofern zu undifferenziert, als es weder die demokratietheoretische Funktion der Verfassung und der Verfassungsgerichtsbarkeit ausreichend reflektiert noch Kriterien dafür angibt, welche Fragen von den politischen und welche von den rechtsstaatlichen Instanzen zu bearbeiten sind. Der Vorwurf gen Karlsruhe, das Gericht habe fälschlicherweise eine ,politische Frage' an sich gerissen und sei dadurch zum "Gegenspieler“ geworden, ist zwar beliebt - er krankt aber daran, dass meist unbestimmt bleibt, was denn eine "politische“ und was eine „rechtliche“ Frage ist. ${ }^{11}$

Aus demokratietheoretischer Sicht kann die notwendige Abgrenzung von politischem und juristischem Handlungsraum über die Funktion der Verfassungsgerichtsbarkeit im demokratischen Verfassungsstaat erfolgen (siehe zu den Funktionen allgemein Grimm 1977; Cardoso da Costa 1988; Brohm 2001; Kranenpohl 2004). Idealerweise soll das Bundesverfassungsgericht dafür sorgen, dass „sich die übrigen Staatsorgane so verhalten, wie sie sich von Verfassungs wegen ohnedies verhalten müßten“, wie Dieter Grimm so treffend formuliert hat. Damit, so Grimm weiter, sei kein Machtverlust der Politik verbunden, sondern nur der „Verlust der Macht, ungestraft gegen die Verfassung zu verstoßen“ (Grimm 1977, S. 87). Wann aber verstoßen politische Akteure gegen die Verfassung? Immer dann, wenn das Verfassungsgericht dies so feststellt?

Dieser Schluss ist empirisch zweifellos richtig, theoretisch aber doch unbefriedigend. Denn natürlich ist ohne weiteres denkbar, dass ein Verfassungsgericht seinen Einfluss überdehnt und sich selbst illegitimerweise autoritativ als - von den Bürgern weder gewählte noch bestätigte - letzte Instanz in allen Dingen einsetzt. Der Ausweg aus diesem Dilemma liegt in einer demokratiefunktionalen Betrachtung der Verfassungsgerichtsbarkeit. Es ist danach zu fragen, in welchen Fällen demokratietheoretisch betrachtet - die Verfassungsgerichtsbarkeit eine Funktion erfüllt, die von politischen Instanzen aufgrund der ihnen eigenen Funktionslogik nicht erbracht werden kann. Hierzu zählt zunächst die Regelung von Kompetenzkonflikten, vor allem aber der Schutz der Grundrechte und der fundamentalen

11 Vorschläge, welche Kriterien bezüglich des Abgrenzungsproblems zwischen Gesetzgeber und Gerichtsbarkeit herangezogen werden können, sind bereits an anderer Stelle gemacht worden (siehe z. B. Schuppert 1980, 1988; Hesse 1981; Roellecke 1987; Kneip 2006). 
Verfassungsprinzipien. Einleuchtend ist zunächst, dass Kompetenzstreitigkeiten zwischen zwei (oder mehr) Akteuren sinnvollerweise von einer dritten, unabhängigen und neutralen Instanz entschieden werden sollten, da sich andernfalls die Mehrheitsposition immer wird durchsetzen können (,Schiedsrichterfunktion' der Verfassungsgerichtsbarkeit). Doch auch die Entscheidung über die Geltung und Reichweite von Grundrechten kann nicht kontingenten politischen Mehrheiten überlassen bleiben. Stünden Grundrechte prinzipiell einer politischen Mehrheit ,zur Verfügung', hätten sie ihren Status als Grundrechte bereits eingebüßt. ${ }^{12} \mathrm{Da}-$ mit mag im Einzelfall zwar immer noch strittig bleiben, wann von einer konkreten politischen Entscheidung oder Handlung in welchem Maße welche Grundrechte betroffen sind - die Verfassungsgerichtsbarkeit ist aber als der Ort bestimmt, an dem legitimerweise über die Geltung der Grundrechte gestritten werden kann und muss.

Aus dieser demokratiefunktionalen Betrachtungsweise entspringt eine weitere wichtige Schlussfolgerung: Wenn es die Aufgabe von Verfassungsgerichten ist, spezifische Funktionen für die Demokratie zu übernehmen, kann nicht mehr pauschal behauptet werden, ein judicial self-restraint wäre per se gut für demokratisches Regieren. Im Gegenteil: Richterliche Zurückhaltung könnte sogar ausgesprochen negativ auf die Demokratie wirken, wenn durch diese Zurückhaltung verfassungswidrige Gesetze oder Handlungen unbeanstandet blieben. ${ }^{13}$ Sinnvollerweise muss daher zwischen vier Kategorien verfassungsgerichtlichen Agierens unterschieden werden: funktionalen Interventionen und Nicht-Interventionen auf der einen und dysfunktionalen Interventionen und Nicht-Interventionen auf der anderen Seite (vgl. Kneip 2006).

Wie sieht es nun mit der konkreten Funktionserfüllung des Bundesverfassungsgerichts aus? Hat es die ihm - verfassungsrechtlich und demokratietheoretisch zugewiesenen Funktionen erfüllen können?

Eine Analyse der 1.297 zwischen 1951 und 2005 in der amtlichen Entscheidungssammlung veröffentlichten Verfahren mit impliziter oder expliziter Normprüfung legt zumindest nahe, dass ihm dies recht gut geglückt zu sein scheint. Um die Entscheidungen des Gerichts zu kategorisieren, wurden sie einem inhaltsanalytischen 4-Stufen-Test unterzogen. Dieser untersuchte, ob a) in den zu entscheidenden Fällen eindeutig formulierte Verfassungsnormen berührt waren, ob b) die zu prüfenden Gesetze oder Handlungen zentrale Grundrechte und Kernbereiche der Demokratie betrafen (z. B. Fragen des Wahlrechts, politische Teilhaberechte, bürgerliche Freiheitsrechte oder die Mechanismen horizontaler Gewaltenkontrolle; vgl. zu den Kerninstitutionen demokratischer Regime z. B. Merkel 2010, S.

12 Damit wird nicht argumentiert, dass politische Mehrheiten Grundrechte grundsätzlich nicht beachten oder garantieren könnten. Allerdings liegt der Sinn von Grundrechtsnormen ja gerade darin, den Gesetzgeber zu binden; er soll gerade nicht mit einfacher Mehrheit die Geltung von Grundrechtsnormen aufheben oder ändern können (vgl. zur Kollision zwischen Grundrechten und Demokratieprinzip z. B. Alexy 1994, S. 407-408).

13 Damit wird auch deutlich, weshalb die Forderung nach ,richterlicher Zurückhaltung' nicht zu analytischer Klarheit beiträgt und letztlich am Problem vorbei zielt: Judicial self-restraint ist keine Frage des richtigen richterlichen Rollenbildes, sondern ein rechtliches oder demokratisches Funktionserfordernis (vgl. hierzu auch Grimm 1976). 
30-37), ob c) ein Agieren des Gesetzgebers im Bereich dieser Grundrechte (Einschränkung oder Ausbau von Rechten) nachvollziehbar (z. B. unter Bezugnahme auf Verhältnismäßigkeitsgesichtspunkte) begründet wurde und ob sich d) die Kontrolldichte des Bundesverfassungsgerichts an demokratietheoretischen Funktionsüberlegungen orientierte. ${ }^{14}$ Legt man diese Kriterien und Codierungen zugrunde, ergibt eine Auswertung der Urteile folgendes Bild: Der weitaus größte Posten der untersuchten Fälle lässt sich unter der Kategorie „funktionale NichtIntervention" fassen (66 Prozent der Fälle, $\mathrm{n}=853$ ). Dies verdeutlicht nochmals die oben getroffene Aussage, dass das Bundesverfassungsgericht in vielen Fällen gar nicht erst in den demokratischen Prozess interveniert, sondern Gesetzesnormen nach Prüfung völlig unbeanstandet lässt. 28 Prozent der untersuchten Fälle $(n=362)$ ließen sich der Kategorie „funktionale Intervention“ zuordnen, betrafen also Fälle, in denen das Bundesverfassungsgericht - nach den hier aufgestellten Kriterien - zu Recht in die Gesetzgebungstätigkeit intervenierte. Die dysfunktionale Kategorie hingegen ist vergleichsweise schwach besetzt: Fünf Prozent der untersuchten Verfahren lassen sich als dysfunktionale Interventionen kategorisieren $(n=69)$ und nur etwa ein Prozent als dysfunktionale Nicht-Interventionen $(\mathrm{n}=13)$; mit der ersten Variante sind solche Fälle gemeint, in denen das Bundesverfassungsgericht nach den oben diskutierten Kriterien nicht hätte eingreifen dürfen, es aber doch getan hat; die zweite Variante meint solche Fälle, in denen es eigentlich hätte eingreifen müssen, es aber nicht getan hat. Insgesamt agiert das Bundesverfassungsgericht damit weitgehend funktional im Sinne der ihm zugewiesenen demokratischen Aufgaben - und dies über alle untersuchten Politikfelder hinweg (vgl. Kneip 2009, S. 315).

14 Die konkrete Codierung der Urteile verlief nach folgenden Kriterien: Verstieß eine Gesetzesnorm eindeutig gegen eine unumstrittene Verfassungsnorm, wurde ein Einschreiten des Bundesverfassungsgerichts als funktional gewertet. Musste die Verfassungsnorm durch das BVerfG interpretiert werden (was in den meisten Verfahren der Fall war), wurde analysiert, ob das Urteil den Grundrechtsbereich im engeren demokratietheoretischen Sinne (Wahlrecht, politische und bürgerliche Rechte, etc.) und/oder weitere Grundprinzipien der Rechtsstaatlichkeit betraf (Rechtsgleichheit, Verhältnismäßigkeitsgrundsatz, Willkürverbot, etc.). War dies der Fall und hielt sich das Gericht an weithin akzeptierte Grundsätze zur Überprüfung von Grundrechtseingriffen (insb. die Prüfung der Verhältnismäßigkeit [Zweck, Geeignetheit, Erforderlichkeit und Angemessenheit einer Regelung]), wurde die Entscheidung als funktional kategorisiert, andernfalls als dysfunktional.

Striktere Kategorisierungsregeln können abstrakt weder demokratietheoretisch noch verfassungsrechtlich abgeleitet (oder gar angewendet) werden; somit wohnt der Kategorisierung funktional/ dysfunktional - anders als jener zwischen Intervention und Nicht-Intervention - womöglich ein Rest von Subjektivität inne. Dieses Problem kann letztlich nur thematisiert und seine Lösung im Einzelfall argumentativ verteidigt werden. Um dies an einem aktuellen Beispiel zu verdeutlichen: In seinem im Februar 2010 ergangenen „Hartz-IV-Urteil“ (1 BvL 1/09 vom 9.02.2010) intervenierte das Bundesverfassungsgericht in einen Politikbereich (Sozialpolitik), der nicht sofort zu den oben skizzierten demokratietheoretischen Kernbereichen zu zählen ist und der auch verfassungsrechtlich vergleichsweise unbestimmt ist. Die Frage, ob sich das BVerfG in dieser Frage hätte - demokratiefunktional - zurückhalten und die Entscheidung des Gesetzgebers akzeptieren sollen, ist also nicht von vornherein abwegig. Allerdings nimmt das Gericht in seinem Urteil eine dezidiert demokratiefunktionale Sichtweise ein, indem es die Gewährung eines ausreichenden Existenzminimums direkt an die Fähigkeit eines Bürgers zur Teilhabe an demokratischen und gesellschaftlichen Prozessen knüpft (Grundrecht auf ein „Mindestmaß an Teilhabe am gesellschaftlichen, kulturellen und politischen Leben “, 1. Leitsatz). Nach den hier diskutierten Kriterien hätte das Urteil daher entgegen des ersten Anscheins - als demokratiefunktional kategorisiert werden müssen. 
Um dies nochmals an Beispielen zu verdeutlichen: Die oben angeführten Annullierungen rechtsstaatseinschränkender Normen des Bundes und der Länder (z. B. Großer Lauschangriff, Luftsicherheitsgesetz, Online-Durchsuchung, vgl. Abschnitt 3.2) können als demokratiefunktionale Interventionen des Bundesverfassungsgerichts kategorisiert werden, weil das Gericht hier in einen Bereich der rechtsstaatlichen Demokratie intervenierte, in dem ihm aus demokratie- und rechtstheoretischer Perspektive eine zentrale Funktion zukommt: der Schutz der Grund- und Bürgerrechte. ${ }^{15}$ Umgekehrt können manche der Interventionen in der Steuer- und Finanzpolitik als dysfunktional kategorisiert werden, da etwa die konkrete Festlegung der Höhe des Existenzminimums nicht zu jenen demokratietheoretischen Funktionen und Aufgaben gehört, die in der Demokratie nur - oder auch nur primär - von der Verfassungsgerichtsbarkeit erbracht werden könnten oder sollten. ${ }^{16}$

Die qualitative Verortung des Urteilsoutputs des Bundesverfassungsgerichts kommt damit zu dem Ergebnis, dass das Gericht mitunter vielleicht ein Gegenspieler von Legislative und Exekutive gewesen ist, nicht aber ein Gegenspieler der Demokratie selbst. Es hat zwar in etwa einem Drittel aller analysierten Verfahren interveniert und Normen des Gesetzgebers beanstandet; mit seinen Interventionen (und Nicht-Interventionen) hat es aber zugleich die demokratische Verfassungsordnung gestützt und die Qualität der Demokratie erhöht. Nochmals mit Dieter Grimm gesprochen hat es zwar in den Interventionsfällen für einen Machtverlust der Staatsorgane gesorgt - diese Macht war aber durch die Verfassung (und den darin kondensierten Volkswillen) ohnehin nicht gedeckt. Die Befürchtung, das Agieren des Gerichts habe die Demokratie geschwächt, kann damit ebenso zurückgewiesen werden wie die Annahme, Interventionen des Gerichts in die Politik seien überhaupt an der Tagesordnung.

15 Die im Jahr 1996 ergangenen Beschlüsse zum sog. „Asylkompromiss“, den das Bundesverfassungsgericht im Kern billigte, können als Beispiele für dysfunktionale Nicht-Interventionen begriffen werden (vgl. BVerfGE 94, 49; 94, 115; 94, 166). Das BVerfG ist mit diesen Beschlüssen seinem Auftrag, zentrale Grundrechte zu schützen, nach den hier diskutierten Kriterien nur unzureichend gerecht geworden. Die Motive des Gerichts für die - für die Fachwelt durchaus überraschende Nicht-Intervention mögen in dem Ziel gelegen haben, das Gericht nach den kurz zuvor ergangenen kontroversen „Soldaten-sind-Mörder“- und „Kruzifix“-Beschlüssen, die dem Gericht einen erheblichen Ansehensverlust in der öffentlichen Meinung beschert hatten, aus der politischen Schusslinie zu nehmen (vgl. hierzu auch Lamprecht 1996; Wesel 2004, S. 326) und so langfristig die eigene Machtposition zu sichern. Nicht-Interventionen (wie Interventionen) können also durchaus für den eigenen langfristigen Machterhalt und in diesem Sinne strategisch eingesetzt werden (ich danke einer anonymen Gutachterin/einem anonymen Gutachter für diesen Hinweis). Die Frage der demokratischen Funktionalität oder Dysfunktionalität von (Nicht-)Interventionen ist jedoch unabhängig von dieser möglichen Motivlage zu beurteilen.

16 Dass dem Schutz der Bürgerrechte hohe Priorität eingeräumt wird und die Festlegung des Existenzminimums als Aufgabe der Politik angesehen wird, liegt nicht zuletzt am hier gewählten Demokratiemodell der rechtsstaatlichen, liberalen Demokratie. Andere Demokratiekonzeptionen kämen möglicherweise zu einer anderen Kategorisierung. Allerdings bekennen sich heute, wie eingangs dargelegt, nahezu alle demokratischen Verfassungen und Gesellschaften zu einem unbedingten Schutz der Grundrechte (und zu einem größeren politischen Handlungsspielraum in sozioökonomischen Fragen), sodass das gewählte Demokratiemodell eine gewisse empirische Relevanz für sich beanspruchen darf. 


\section{Fazit: Gegenspieler? Vetospieler? Mitspieler!}

Der vorliegende Beitrag hat sich dreier zusammenhängender Fragen gewidmet. Erstens hat er danach gefragt, ob und inwiefern Verfassungsgerichte als Vetooder Gegenspieler der Politik begriffen werden können und inwiefern ihr Agieren zu einer Justizialisierung demokratischer politischer Prozesse führt. Zweitens hat er sich der Frage gewidmet, welche Folgen das Agieren von Verfassungsgerichten für demokratisches Regieren hat, und einen Vorschlag unterbreitet, wie ,gutes oder schlechtes Agieren' über einen demokratietheoretischen Zugang operationalisiert und gemessen werden kann. Drittens schließlich hat er durch eine empirische Analyse des Urteilsoutputs des Bundesverfassungsgerichts zu zeigen versucht, dass zumindest in den letzten 60 Jahren Karlsruhe nicht als ausgeprägter Vetospieler in Erscheinung getreten ist und auch keine prinzipielle Gegenspielerposition zur Politik eingenommen hat - was selbstverständlich nicht ausschließt, dass dies in Einzelfällen doch einmal so gewesen ist.

Mit der vorgenommenen empirischen Outputanalyse des Bundesverfassungsgerichts konnte die Befürchtung ausgeräumt werden, dass verfassungsgerichtliches Agieren per se zu zunehmender Justizialisierung der Politik führt. Obwohl das Bundesverfassungsgericht Jahr um Jahr mit immer mehr Klagen aus Politik, Gerichtswesen und Bevölkerung belastet wird, hat es keineswegs immer häufiger in die Politik hineinregiert. Im Gegenteil, seine Annullierungsquoten blieben über die Jahre bemerkenswert konstant.

Betrachtet man schließlich die Urteile des Bundesverfassungsgerichts auch noch unter dem demokratietheoretisch wichtigen Gesichtspunkt ihrer Demokratiefunktionalität, muss der skeptische Blick auf die Verfassungsgerichtsbarkeit sogar einem weitaus optimistischeren weichen. Unter funktionalen Gesichtspunkten ist die Leistungsbilanz des Bundesverfassungsgerichts nämlich nochmals höher zu bewerten. Die Karlsruher Richterinnen und Richter haben in insgesamt nur sehr wenigen Fällen ihre funktionale Rolle im Gewaltenteilungssystem dahingehend überdehnt, dass sie Dinge entschieden hätten, zu denen sie aus demokratietheoretischer Sicht nicht befugt waren. Diese Ergebnisse sollten Anlass sein, die Sichtweise auf die demokratische Verfassungsgerichtsbarkeit zu überdenken. Begreift man „Demokratie“ als rechts- und verfassungsstaatliche Demokratie, in der die Geltung von Grund- und Bürgerrechten der Verfügungsgewalt kontingenter politischer Mehrheiten legitimerweise entzogen ist, dann sind Verfassungsgerichte keine prinzipiellen Veto- oder Gegenspieler der Politik, sondern legitime Mitspieler in der Demokratie. Als Mitspieler zeichnen sie sich dadurch aus, dass sie - zusammen mit den anderen Spielern und nicht gegen sie - an einem gemeinsamen Ziel arbeiten: der adäquaten Ausgestaltung und Interpretation der Verfassungsordnung als Erst- bzw. Zweitinterpreten. Dies bedeutet freilich nicht, dass verfassungsgerichtliche Urteile durch Politik, Wissenschaft und Gesellschaft nicht aufmerksam begleitet werden müssten. Ganz im Gegenteil: Aufgabe insbesondere der politischen Instanzen muss es sein, das Agieren der Verfassungsgerichtsbarkeit kritisch zu begleiten und sie immer wieder daran zu erinnern, dass ihr zwar im demokratischen Verfassungsstaat das letzte Wort zukommt, dass dieses aber nur dann dauerhaft Legitimität beanspruchen kann, wenn die demokratiefunktiona- 
len Grenzen verfassungsgerichtlichen Agierens Leitschnur ihres Handelns bleiben. Dem Bundesverfassungsgericht ist dies in den letzten Jahrzehnten vergleichsweise gut geglückt. Vergleichende Studien anderer Verfassungsgerichte müssen erst zeigen, ob dies als generelle Erkenntnis gelten darf.

\section{Literatur}

Abromeit, Heidrun. 1999. Volkssouveränität in komplexen Gesellschaften. In Das Recht der Republik, Hrsg. Hauke Brunkhorst und Peter Niesen, 17-36. Frankfurt a. M.: Suhrkamp.

Alexander, Larry (Hrsg.). 1998. Constitutionalism. Philosophical Foundations. Cambridge: Cambridge University Press.

Alexy, Robert. 1994. Theorie der Grundrechte. Frankfurt a. M.: Suhrkamp.

Alivizatos, Nicos C. 1995. Judges as Veto Players. In Parliaments and Majority Rule in Western Europe, Hrsg. Herbert Döring, 566-589. Frankfurt/New York: Campus Verlag und St. Martin's Press.

Bellamy, Richard (Hrsg.). 1996. Constitutionalism, Democracy and Sovereignty: American and European Perspectives. Aldershot: Avebury.

Benda, Ernst. 1979. Bundesverfassungsgericht und Gesetzgeber im dritten Jahrzehnt des Grundgesetzes. Die öffentliche Verwaltung 32:465-470.

Beyme, Klaus von. 1985. Verfassungsgerichtsbarkeit und Policy-Analysis. In Festschrift für Rudolf Wassermann zum sechzigsten Geburtstag, Hrsg. Christian Broda et al., 259278. Neuwied: Luchterhand.

Beyme, Klaus von. 1997. Der Gesetzgeber. Der Bundestag als Entscheidungszentrum. Wiesbaden: Westdeutscher Verlag.

Blasberg, Georg. 2003. Verfassungsgerichte als Ersatzgesetzgeber. Entscheidungsaussprüche bei Normenkontrollen von Bundesverfassungsgericht und Corte Costituzionale. Frankfurt a. M.: Peter Lang.

Brohm, Winfried. 2001. Die Funktion des BVerfG - Oligarchie in der Demokratie? Neue Juristische Wochenschrift 54:1-10.

Cardoso da Costa, Jose Manuel Moreira. 1988. Die Verfassungsrechtsprechung im Rahmen der staatlichen Funktionen. Arten, Inhalt und Wirkungen der Entscheidungen über die Verfassungsmäßigkeit von Rechtsnormen. Generalbericht. Europäische Grundrechte-Zeitschrift 15:236-248.

Elster, Jon, und Rune Slagstad (Hrsg.). 1993. Constitutionalism and Democracy. Cambridge: Cambridge University Press.

Epstein, Lee, und Jack Knight. 2004. Courts and Judges. In The Blackwell Companion to Law and Society, Hrsg. Austin Sarat, 170-194. Oxford: Blackwell.

Epstein, Lee, Jack Knight, und Olga Shvetsova. 2001. The Role of Constitutional Courts in the Establishment and Maintenance of Democratic Systems of Government. Law \& Society Review 35:117-163.

Gawron, Thomas, und Ralf Rogowski. 2007. Die Wirkung des Bundesverfassungsgerichtes. Rechtssoziologische Analysen. Baden-Baden: Nomos.

Ginsburg, Tom. 2003. Judicial Review in New Democracies. Constitutional Courts in Asian Cases. Cambridge: Cambridge University Press. 
Grimm, Dieter. 1976. Verfassungsgerichtsbarkeit im demokratischen System. Juristenzeitung 31:697-703.

Grimm, Dieter. 1977. Verfassungsgerichtsbarkeit - Funktion und Funktionsgrenzen im demokratischen Staat. In Sozialwissenschaften im Studium des Rechts, Hrsg. Wolfgang Hoffmann-Riem, 83-108. München: C. H. Beck.

Guarnieri, Carlo, und Patrizia Pederzoli. 2002. The Power of Judges. A Comparative Study of Courts and Democracy. Oxford: Oxford University Press.

Haley, John O. 2005. The Japanese Judiciary: Maintaining Integrity, Autonomy and the Public Trust. Washington: Washington University School of Law.

Henkin, Louis. 1994. A New Birth of Constitutionalism: Genetic Influences and Genetic Defects. In Constitutionalism, Identity, Difference, and Legitimacy, Hrsg. Michael Rosenfeld, 39-52. Durham: Duke University Press.

Hesse, Konrad. 1981. Funktionelle Grenzen der Verfassungsgerichtsbarkeit. In Recht als Prozess und Gefüge. Festschrift für Hans Huber zum 80. Geburtstag, Hrsg. Kurt Eichenberger und Jörg Paul Müller, 261-272. Bern: Stämpfli.

Hesse, Konrad. 1999 [1966]. Grundzüge des Verfassungsrechts der Bundesrepublik Deutschland. Heidelberg: C. F. Müller.

Hilbink, Lisa. 2008. Assessing the New Constitutionalism. Comparative Politics 40:227245.

Hirschl, Ran. 2007. Towards Juristocracy. The Origins and Consequences of the New Constitutionalism. Cambridge: Harvard University Press.

Höffe, Otfried. 1999. Wieviel Politik ist dem Verfassungsgericht erlaubt? Der Staat 38:171193.

Holmes, Stephen. 1995. Constitutionalism. In The Encyclopedia of Democracy, Hrsg. Seymour Martin Lipset, 299-306. London: Taylor \& Francis.

Hönnige, Christoph. 2007. Verfassungsgericht, Regierung und Opposition. Die vergleichende Analyse eines Spannungsdreiecks. Wiesbaden: VS Verlag für Sozialwissenschaften.

Hönnige, Christoph, und Thomas Gschwend. 2010. Das Bundesverfassungsgericht im politischen System der BRD - ein unbekanntes Wesen? Politische Vierteljahresschrift 51:507-530.

Horowitz, Donald L. 2006. Constitutional Courts: A primer for decision makers. Journal of Democracy 17:125-137.

Hufen, Friedhelm. 1998. Anbringen von Kruzifixen in staatlichen Schulen als Verstoß gegen Art. 4 Abs. 1 GG. In Hüter der Verfassung oder Lenker der Politik? Das Bundesverfassungsgericht im Widerstreit, Hrsg. Bernd Guggenberger und Thomas Würtenberger, 161-172. Baden-Baden: Nomos.

Itoh, Hiroshi. 1990. Judicial Review and Judicial Activism in Japan. Law and Contemporary Problems 53:169-179.

Jäger, Wolfgang. 1986. Die Innenpolitik der sozial-liberalen Koalition 1969-1974. In Geschichte der Bundesrepublik Deutschland. Band 5: Republik im Wandel. Die Ära Brandt, Hrsg. Karl-Dietrich Bracher, Wolfgang Jäger und Werner Link, 15-160. Stuttgart: Deutsche Verlags-Anstalt.

Kavanagh, Aileen. 2009. Constitutional Review under the UK Human Rights Act. Cambridge: Cambridge University Press. 
Kirchhof, Paul. 1998. Verfassungsgerichtsbarkeit und Gesetzgebung. In Verfassungsgerichtsbarkeit und Gesetzgebung. Symposion aus Anlaß des 70. Geburtstages von Peter Lerche, Hrsg. Peter Badura und Rupert Scholz, 5-22. München: C. H. Beck.

Kneip, Sascha. 2006. Demokratieimmanente Grenzen der Verfassungsgerichtsbarkeit. In Politik und Recht. PVS-Sonderheft 36/2006, Hrsg. Michael Becker und Ruth Zimmerling, 259-281. Wiesbaden: VS Verlag für Sozialwissenschaften.

Kneip, Sascha. 2009. Verfassungsgerichte als demokratische Akteure. Der Beitrag des Bundesverfassungsgerichts zur Qualität der bundesdeutschen Demokratie. Baden-Baden: Nomos.

Koopmans, Tim. 2003. Courts and Political Institutions. A Comparative View. Cambridge: Cambridge University Press.

Köppe, Olaf. 2006. Bundesverfassungsgericht und Steuergesetzgebung - Politik mit den Mitteln der Verfassungsrechtsprechung? In Das Bundesverfassungsgericht im politischen Prozess, Hrsg. Robert Chr. Van Ooyen und Martin H. W. Möllers, 435-447. Wiesbaden: VS Verlag für Sozialwissenschaften.

Kranenpohl, Uwe. 2004. Funktionen des Bundesverfassungsgerichts. Eine politikwissenschaftliche Analyse. Aus Politik und Zeitgeschichte B50-51:39-46.

Kranenpohl, Uwe. 2010. Hinter dem Schleier des Beratungsgeheimnisses: Der Willensbildungs- und Entscheidungsprozess des Bundesverfassungsgerichts. Wiesbaden: VS Verlag für Sozialwissenschaften.

Lamprecht, Rolf. 1996. Zur Demontage des Bundesverfassungsgerichts. Beweissicherung und Bestandsaufnahme. Baden-Baden: Nomos.

Landfried, Christine. 1984. Bundesverfassungsgericht und Gesetzgeber. Wirkungen der Verfassungsrechtsprechung auf parlamentarische Willensbildung und soziale Realität. Baden-Baden: Nomos.

Landfried, Christine. 1985. The Impact of the German Federal Constitutional Court on Politics and Policy Output. Government and Opposition 20:522-541.

Landfried, Christine. 1992. Judicial Policy-Making in Germany: The Federal Constitutional Court. West European Politics 15:50-67.

Landfried, Christine. 1994. The Judicialization of Politics in Germany. International Political Science Review 15:113-124.

Lembcke, Oliver. 2007. Hüter der Verfassung: Eine institutionentheoretische Studie zur Autorität des Bundesverfassungsgerichts. Tübingen: Mohr Siebeck.

Lhotta, Roland. 2003. Das Bundesverfassungsgericht und die „Generationengerechtigkeit“. In Sozial- und Wirtschaftspolitik unter Rot-Grün, Hrsg. Antonia Gohr und Martin Seeleib-Kaiser, 307-327. Wiesbaden: Westdeutscher Verlag.

Lijphart, Arend. 1999. Patterns of Democracy. Government Forms and Performance in Thirty-Six Countries. New Haven: Yale University Press.

Magaloni, Beatriz, und Arianna Sánchez. 2001. Empowering Courts as Constitutional Veto Players: Presidential Delegation and the New Mexican Supreme Court. Beitrag präsentiert auf dem Annual Meeting of the American Political Science Association, 2001, San Francisco.

Maus, Ingeborg. 1991. Sinn und Bedeutung von Volkssouveränität in der modernen Gesellschaft. Kritische Justiz 24:137-150.

Maus, Ingeborg. 1992. Zur Aufklärung der Demokratietheorie. Rechts- und demokratietheoretische Überlegungen im Anschluß an Kant. Frankfurt a. M.: Suhrkamp. 
Maus, Ingeborg. 2004. Vom Rechtsstaat zum Verfassungsstaat. Zur Kritik juridischer Demokratieverhinderung. Blätter für deutsche und internationale Politik. 2004: 835850.

Maus, Ingeborg. 2005. Zur Ideengeschichte der Gewaltenteilung und der Funktionsweise der Justiz. In Demokratie in Europa und europäische Demokratien. Festschrift für Heidrun Abromeit, Hrsg. Tanja Hitzel-Cassagnes und Thomas Schmidt, 224-262, Wiesbaden: VS Verlag für Sozialwissenschaften.

Merkel, Wolfgang. 2010. Systemtransformation. Eine Einführung in die Theorie und Empirie der Transformationsforschung. Wiesbaden: VS Verlag für Sozialwissenschaften.

Mückl, Stefan. 2005. Kooperation oder Konfrontation? Das Verhältnis zwischen Bundesverfassungsgericht und Europäischem Gerichtshof für Menschenrechte. Der Staat 44:403-431.

Ooyen, Robert Chr. van, und Martin H. W. Möllers (Hrsg.). 2006. Das Bundesverfassungsgericht im politischen System. Wiesbaden: VS Verlag für Sozialwissenschaften.

Ramseyer, J. Mark. 1991. The Japanese Supreme Court: Constitutional Policies. Journal of Japanese Studies 17:176-178.

Ramseyer, J. Mark, und Eric B. Rasmusen. 2003. Measuring Judicial Independence. The Political Economy of Judging in Japan. Chicago: The University of Chicago Press.

Roellecke, Gerd. 1987. Aufgaben und Stellung des Bundesverfassungsgerichts im Verfassungsgefüge. In Handbuch des Staatsrechts der Bundesrepublik Deutschland. Band II, Hrsg. Josef Isensee und Paul Kirchhof, 683-696. Heidelberg: C. F. Müller.

Romeu, Francisco Ramos. 2006. The Establishment of Constitutional Courts: A Study of 128 Democratic Constitutions. Review of Law and Economics 2:104-135.

Sandler, Ross, und David Schoenbrod. 2003. Democracy by Decree: What Happens When Courts Run Government. New Haven: Yale University Press.

Schaal, Gary S. 2006. Crisis! What Crisis? Der „Kruzifix-Beschluss“ und seine Folgen. In Das Bundesverfassungsgericht im politischen System, Hrsg. Robert Chr. van Ooyen und Martin H. W. Möllers, 175-186. Wiesbaden: VS Verlag für Sozialwissenschaften.

Scheppele, Kim Lane. 2005. Democracy by Judiciary. Or, why Courts Can be More Democratic than Parliaments. In Rethinking the Rule of Law after Communism, Hrsg. Adam Czarnota, Martin Krygier und Wojciech Sadurski, 26-60. Budapest/New York: Central European University Press.

Schlaich, Klaus, und Stefan Korioth. 2004. Das Bundesverfassungsgericht. Stellung, Verfahren, Entscheidungen. München: C. H. Beck.

Schmidt, Manfred G. 2002. Germany: The Grand Coalition State. In Political Institutions in Europe, Hrsg. Josep M. Colomer, 55-93. London: Routledge.

Schmidt, Torsten. 2001. Finanzreformen in der Bundesrepublik Deutschland. Analyse der Veränderungen der Finanzverfassung von 1949 bis 1989. Berlin: Duncker \& Humblot.

Schneider, Hans-Peter. 1999. Acht an der Macht! Das Bundesverfassungsgericht als „Reparaturbetrieb“ des Parlamentarismus? Neue Juristische Wochenschrift 52:13031305 .

Scholz, Rupert. 1999. Das Bundesverfassungsgericht: Hüter der Verfassung oder Ersatzgesetzgeber? Aus Politik und Zeitgeschichte B16:3-8.

Schuppert, Gunnar Folke. 1980. Funktionell-rechtliche Grenzen der Verfassungsinterpretation. Königstein: Athenaeum. 
Schuppert, Gunnar Folke. 1988. Self-restraints der Rechtsprechung. Überlegungen zur Kontrolldichte in der Verfassungs- und Verwaltungsgerichtsbarkeit. Deutsches Verwaltungsblatt 103:1191-1200.

Shapiro, Martin, und Alec Stone Sweet. 2002. On Law, Politics, and Judicialization. Oxford: Oxford University Press.

Steinsdorff, Silvia von. 2009. Das Verfahren zur Rekrutierung der Bundesverfassungsrichter: Reformbedürftige Schwachstelle eines Grundpfeilers der politischen Ordnung. In Ordnung und Wandel als Herausforderungen für Staat und Gesellschaft. Festschrift für Gert-Joachim Glaeßner, Hrsg. Astrid Lorenz und Werner Reutter, 279-306. Opladen/ Farmington Hills: Barbara Budrich.

Stone, Alec. 1992. The Birth of Judicial Politics in France. The Constitutional Council in Comparative Perspective. Oxford: Oxford University Press.

Stone Sweet, Alec. 1999. Judicialization and the Construction of Governance. Comparative Political Studies 32:147-184.

Stone Sweet, Alec. 2000. Governing with Judges. Constitutional Politics in Europe. Oxford: Oxford University Press.

Stüwe, Klaus. 1997. Die Opposition im Bundestag und das Bundesverfassungsgericht. Das verfassungsgerichtliche Verfahren als Kontrollinstrument der parlamentarischen Minderheit. Baden-Baden: Nomos.

Stüwe, Klaus. 2001. Das Bundesverfassungsgericht als verlängerter Arm der Opposition? Eine Bilanz seit 1951. Aus Politik und Zeitgeschichte B37-38:34-44.

Tate, C. Neal, und Torbjörn Vallinder. 1995. Judicialization and the Future of Politics and Policy. In The Global Expansion of Judicial Power, Hrsg. C. Neal Tate und Torbjörn Vallinder, 515-528. New York: New York University Press.

Tsebelis, George. 1995. Decision Making in Political Systems: Veto Players in Presidentialism, Parliamentarism, Multicameralism and Multipartyism. British Journal of Political Science 25:289-326.

Tsebelis, George. 2002. Veto Players. How Political Institutions Work. Princeton: Princeton University Press.

Vallinder, Torbjörn. 1994. The Judicialization of Politics - A World-wide Phenomenon: Introduction. International Political Science Review 15:91-99.

Vallinder, Torbjörn. 1995. When the courts go marching in. In The Global Expansion of Judicial Power, Hrsg. C. Neal Tate und Torbjörn Vallinder, 13-26. New York: New York University Press.

Volcansek, Mary L. 2001. Constitutional courts as veto players: Divorce and decrees in Italy. European Journal of Political Research 39:347-372.

Vorländer, Hans (Hrsg.). 2006. Die Deutungsmacht der Verfassungsgerichtsbarkeit. Wiesbaden: VS Verlag für Sozialwissenschaften.

Voßkuhle, Andreas. 2000. Theorie und Praxis der verfassungskonformen Auslegung von Gesetzen durch Fachgerichte. Kritische Bestandsaufnahme und Versuch einer Neubestimmung. Archiv des öffentlichen Rechts 125:177-201.

Wagschal, Uwe. 2006. Entwicklung, Determinanten und Vergleich der Staatsfinanzen. In Regieren in der Bundesrepublik Deutschland. Innen- und Außenpolitik seit 1949, Hrsg. Manfred G. Schmidt und Reimut Zohlnhöfer, 57-85. Wiesbaden: VS Verlag für Sozialwissenschaften. 
Wagschal, Uwe. 2006. Verfassungsgerichte als Vetospieler in der Steuerpolitik. In Politik und Recht. PVS-Sonderheft 36/2006, Hrsg. Michael Becker und Ruth Zimmerling, 559-584. Wiesbaden: VS Verlag für Sozialwissenschaften.

Waldron, Jeremy. 1999. Law and Disagreement. Oxford: Clarendon Press.

Waldron, Jeremy. 2006. The Core of the Case Against Judicial Review. Yale Law Journal 115:1346-1406.

Waltman, Jerold L., und Kenneth M. Holland (Hrsg.). 1988. The Political Role of Law Courts in Modern Democracies. New York: St. Martin's Press.

Wesel, Uwe. 2004. Der Gang nach Karlsrube. Das Bundesverfassungsgericht in der Geschichte der Bundesrepublik. München: Blessing.

Zippelius, Reinhold. 1976. Verfassungskonforme Auslegung von Gesetzen. In Bundesverfassungsgericht und Grundgesetz. Festgabe aus Anlaß des 25jährigen Bestehens des Bundesverfassungsgerichts. Zweiter Band. Verfassungsauslegung, Hrsg. Christian Starck, 108-124. Tübingen: Mohr Siebeck.

\section{Autorenangaben}

Dr. Sascha Kneip,

Wissenschaftszentrum Berlin für Sozialforschung, Abteilung Demokratieforschung,

Reichpietschufer 50, 10785 Berlin,

kneip@wzb.eu 